\title{
Jugendstrafrecht im europäischen Vergleich im Licht aktueller Empfehlungen des Europarats
}

Frieder Dünkel

\section{Einleitung}

Jugendkriminalität als international vergleichend gesehen flächendeckende und „ubiquitäre“ Erscheinung weist im Hinblick auf ihre Hintergründe und Auswirkungen häufig Gleichförmigkeiten auf. ${ }^{1}$ Die Jugendstrafrechtssysteme und damit die Reaktionen auf straffälliges Verhalten Minderjähriger unterscheiden sich hingegen deutlich voneinander. Vor allem in den letzten 20 Jahren haben sie einen tief greifenden Wandel erlebt, insbesondere in den Ländern im Übergang von staatsautoritären hin zu demokratisch verfassten Systemen im Einflussbereich der ehemaligen Sowjetunion. Aber auch in Westeuropa gab es weit reichende Reformen, die man in ihrer eher straforientierten Ausrichtung z. B. in England und Wales, aber auch in Frankreich oder den Niederlanden als neo-liberal charakterisiert hat. ${ }^{2}$ In anderen Ländern wie z. B. Belgien oder Deutschland wurde die moderate und vorrangig am Erziehungsgedanken ausgerichtete Jugendkriminalpolitik beibehalten (Vorrang der Diversion und Anwendung des Prinzips „Erziehung statt Strafe“, vgl. Dünkel 2006). In zahlreichen Ländern wurden Elemente einer wiedergutmachenden Strafrechtspflege („restorative justice“) mit Täter-Opfer-Ausgleichsprogrammen, Familiengruppenkonferenzen etc. eingeführt bzw. ausgebaut (z. B. in Belgien und Nordirland, vgl. Dünkel/Grzywa/Pruin 2009).

Fragen, die sich in diesem Zusammenhang stellen, betreffen die zukünftige Orientierung des Jugendstrafrechts in den europäischen Ländern und insbesondere, ob sich die eher angelsächsische, neo-liberale oder die wohlfahrtsorientierte Tendenz durchsetzen wird. Welche Rolle werden dabei Aspekte der wiedergutmachenden Strafrechtspflege und die Berücksichtigung von Opferinteressen spielen? Welche Herangehensweisen im Umgang mit jungen Straftätern haben sich in den einzelnen Ländern als besonders wirksam erwiesen, so dass sich eine Übertragung auf den gesamteuropäischen Raum lohnen könnte? Ferner ist aus Sicht der Europäischen Union ebenso wie des Europarats die Frage legitim, inwieweit eine stärkere Harmonisierung bzw. Berücksichtigung gemeinsamer europäischer Mindeststandards erreicht werden kann.
Obwohl es in den vergangenen Jahren einige (rechts-) vergleichende Analysen des Jugendstrafrechts gab (vgl. Albrecht/Kilchling 2002; Cavadino/Dignan 2006), gibt es doch ein weitgehendes Defizit an Informationen vertiefender und umfassenderer Forschung, die insbesondere auch die mittelund osteuropäischen Länder bzw. die neuen Mitgliedsstaaten der EU bzw. die entsprechenden Beitrittskandidaten einbeziehen. Einige frühere Publikationen wie diejenigen von McCarney (1996), Dünkellvan Kalmthout/Schüler-Springorum (1997) Mehlbyel Walgrave (1998) und Dünkel/Drenkhahn (2003) haben das Jugendstrafrecht einiger dieser Länder beschrieben, sind aber nicht mehr aktuell. Ein Teil der neueren vergleichenden Literatur wie die Sammelbände von Albrecht/Kilchling (2002), Tonry/Doob (2004), Jensen/Jepsen (2006) und JungerTas/Decker (2006) sind stark auf Westeuropa und die USA bzw. Kanada fokussiert, während die mittel- und osteuropäischen Länder nur vereinzelt einbezogen werden. Gleiches gilt für den ersten Sammelband einer italienisch/kroatischen Kooperation (vgl. Patané 2007), der insoweit lediglich Berichte von Kroatien, Litauen und Slowenien enthält. Hinzu kommt, dass die bisher vorliegende Forschung sich schwerpunktmäßig auf die Jugendstrafrechtssysteme beschränkt und den Vollzug von ambulanten und stationären Sanktionen einschließlich des Jugendstrafvollzugs weitgehend ausklammert. In kaum einer der aufgeführten Publikationen wurden die Berichte nach einer einheitlichen Fragestellung verfasst, so dass die Vergleichbarkeit allein aus diesem Grund stark eingeschränkt bleibt. Dies verdeutlicht den Forschungsbedarf hinsichtlich einer umfassenderen und weit mehr Länder erfassenden vergleichenden Analyse. Diesem Anliegen wurde bzw. wird in einem von der EU geförderten AGIS-Projekt "Juvenile Justice Systems in Europe - current situation, reform developments and good practices" (JLS/2006/AGIS/168) Rechnung getragen, das von der Universität Greifswald, Lehrstuhl für Kriminologie koordiniert wird (s. hierzu auch die Informationen auf der Internet-Seite des Lehrstuhls: http://jura.unigreifswald.de/duenkel).
Das bis Mitte 2009 laufende Projekt wird in einem ersten Schritt mit der Veröffentlichung von 33 Landesberichten zum Jugendkriminalrecht in Europa nach einem einheitlichen Gliederungsschema sowie vergleichenden Analysen abgeschlossen werden. In diesem Projekt zeigt sich, dass in Europa teilweise höchst unterschiedliche Modelle zum Umgang mit jungen Straftätern verfolgt werden. Gerade weil sich die Jugendkriminalität in ihrem Umfang und ihrer Struktur und den gesellschaftlichen Ursachen so stark ähnelt, handelt es sich hier um einen Bereich, in dem gut von wechselseitigen Erfahrungen profitiert werden könnte. Diese Erkenntnis legt die Grundlage für den derzeit laufenden zweiten Projektteil, in dem es um die Fokussierung auf „gute Praxismodelle“ des Jugendstrafrechts geht, die im Hinblick auf ihre Evaluation und Übertragbarkeit überprüft werden.

\section{Aktuelle Reformtendenzen der Jugendkriminalpolitik}

Europaweit kann man am Gedanken der Subsidiarität bzw. Verhältnismäßigkeit von staatlichen Eingriffen gegenüber straffälligen Jugendlichen orientierte Tendenzen der Jugendkriminalpolitik erkennen, wie sie auch in den Mindestgrundsätzen der Vereinten Nationen zur Jugendgerichtsbarkeit von 1985 oder der Kinderrechtskonvention von 1989 zum Ausdruck gelangen. Hierbei geht es auch um den Ausbau rechtsstaatlicher Verfahrensgarantien einerseits und die Begrenzung bzw. Reduzierung der Eingriffsintensität im Rechtsfolgenbereich andererseits.

Allerdings sind in jüngerer Zeit in einigen europäischen Ländern auch gegenläufige Tendenzen sichtbar, die Verschärfungen des Jugendstrafrechts mit einer Anhebung der Höchststrafen bei der Jugendstrafe und der Einführung anderer Formen einer sicheren Unterbringung beinhalten. $\mathrm{Zu}$ nennen sind die Jugendstrafrechtsreformen in den Niederlanden 1995 und Frankreich 1996, 2002 bzw. $2007^{3}$ und in England von 1994 bzw. 1998. ${ }^{4}$ Auch in den skandinavischen Ländern, die kein eigenständiges Jugendstrafrecht haben, jedoch durch Verweisungen an die Jugendhilfe (vgl. insbesondere Schweden) ein eher wohlfahrtsorientiertes Modell 
entwickelt haben, ${ }^{5}$ gibt es Tendenzen, vor allem für Rückfalltäter neue Sanktionen zu schaffen. In Dänemark wurde eine spezielle Jugendstrafe eingeführt, die eine Kombination von freiheitsentziehenden und nicht freiheitsentziehenden Elementen während der Dauer von zwei Jahren enthält und die an die Stelle zuvor üblicher kurzer Freiheitsstrafen getreten ist. Schweden hat 1999 die geschlossene Jugendfürsorge (Heimerziehung) ausgebaut und schließlich beinhalten die sog. Vertragsstrafen in Dänemark verbindlichere und eingriffsintensivere Formen der Diversion. Auch Finnland hat 2005 mit der sog. Jugendstrafe (von 4 Monaten bis zu einem Jahr) eine spezifische Sanktionsform für Jugendliche geschaffen und damit den Trend in skandinavischen Ländern bestätigt, trotz Fehlens einer eigenständigen Jugendgerichtsbarkeit spezifisch jugendstrafrechtliche Sanktionsformen zu schaffen bzw. auszuweiten (vgl. Haferkamp 2007, S. 186 f.). Gleichwohl stellen sich die Reformen in den skandinavischen Ländern als eher moderat dar und sind keineswegs dem „neo-liberalen“ Modell englischer Prägung (s. u.) gleichzustellen.

Die Ursachen einer in einigen Ländern zu beobachtenden Verschärfung des Jugendstrafrechts sind vielfältig. Sicherlich ist der allgemeine „punitive“ Trend mit Anleihen aus Vergeltungs- und tatorientierten Strafphilosophien aus den USA nicht ohne Wirkung in Europa geblieben. Die „neue Straflust " 6 macht auch vor dem Jugendstrafrecht nicht halt, wenngleich hier - auch angesichts eindeutiger internationaler Vorgaben (s. u. 4. und 5.) - noch am ehesten repressiven Tendenzen entgegengewirkt werden kann. Straforientierte Konzepte haben in einigen Ländern durch zunehmende Probleme mit Migranten und ethnischen Minderheiten sowie Verwerfungen auf dem Arbeitsmarkt mit einem beachtlichen und zunehmenden Anteil von schlecht ausgebildeten Jugendlichen, die kaum Perspektiven haben, an Bedeutung gewonnen (vgl. Junger-Tas 2006, S. 522 ff.). In diesem Zusammenhang spielt auch eine gewisse Ratlosigkeit im Umgang mit Mehrfachauffälligen eine Rolle. Daher sind Tendenzen der Strafschärfung vielfach auf Mehrfachauffällige bzw. Rückfalltäter begrenzt, wie insbesondere die Entwicklung in Frankreich oder Skandinavien belegt. Die Renaissance geschlossener Heimerziehung ist vor diesem Hintergrund zu sehen.

Neu in der Diskussion zur Reform des Jugendhilfe- bzw. Jugendkriminalrechts ist, die Eltern u. U. auch strafrechtlich verant- wortlich zu machen. Die sog. parenting order in England ermöglicht es, Eltern im Falle fehlender Beaufsichtigung für die Straftaten ihrer Kinder verantwortlich zu machen, indem sie z. B. zur Zahlung einer Geldbuße oder der Teilnahme an einem Elterntraining verpflichtet werden. In Frankreich wurde 2002 die Haftung der Eltern dahingehend verschärft, dass ihnen im Falle der Unterbringung des Kindes bzw. Jugendlichen in einer geschlossenen Einrichtung das Kindergeld gestrichen werden kann. Ferner können sie mit einer Geldbuße belegt werden, wenn sie auf Ladung des Richters zur Verhandlung des Jugendgerichts nicht erscheinen (vgl. Kasten 2003, S. 387).

Das Schlagwort der „Verantwortlichmachung" (responsibility) ist in England zu einer zentralen Kategorie des Jugendstrafrechts geworden (vgl. Graham 1998; kritisch Cavadino/Dignan 2004). Im positiven Sinne ist sie mit den Bestrebungen zur Ausweitung des Täter-Opfer-Ausgleichs, der mediation bzw. der Wiedergutmachung verknüpft, problematisch allerdings ist sie in Verbindung mit der Abschaffung des doli incapax für 10-14-Jährige und damit einer erheblichen Absenkung des Strafmündigkeitsalters. Die jugendkriminalpolitischen Tendenzen in England können als symptomatisch für neo-liberale Orientierungen (die sog. 4 R's) unter den Stichworten responsibility und restitution (reparation), restorative justice, aber auch der (gelegentlich offen propagierten) retribution (Vergeltung), gesehen werden, die die Debatte der 1960er und 1970er Jahre unter den Schlagworten der sog. vier D's (diversion, decriminalization, deinstitutionalization, due process) abgelöst haben (vgl. Dünkel 2003). Die Kategorie der Vergeltung wird z. B. daran deutlich, dass für ambulante Maßnahmen gefordert wird, sie sollten hart (,tough“) und „glaubwürdig“ („credible“) ausgestaltet werden. So wurde aus dem „community treatment“ der 1960er Jahre das „community punishment" der 1980er und 1990er Jahre. Cavadino und Dignan bezeichnen diese Strömungen als das „neo-correctionalist model“ (vgl. Cavadino/Dignan 2006, S. 210 ff. und unten 3.).

Gleichwohl kann man - zumindest für die kontinentaleuropäischen Länder - keinen Rückfall in klassische Auffassungen des 18. und 19. Jahrhunderts feststellen. Überall wird am vorrangigen Prinzip der Erziehung bzw. Spezialprävention festgehalten, auch wenn die Justizorientierung sich verstärkt hat und damit das Spannungsfeld, wenn nicht gar Paradox von Erziehung und Strafe evident bleibt. Als beispielhaft dafür kann man die Reformgesetze in Österreich von 1988 und 2001, in Deutschland von 1990, in den Niederlanden von 1995, in Spanien von 2000, in Portugal von 2001 und in Frankreich und Nordirland von 2002, aber auch in Litauen von 2000 (vgl. hierzu Dünkel/Sakalauskas 2001) und Tschechien von 2003 (vgl. Válková 2006) oder Serbien von 2006 (vgl. Škulić 2009) ansehen (vgl. zusammenfassend Dünkel 2003; Dünkel/ Grzywa/Pruin 2009).

In diesem Zusammenhang lassen sich spezifische erfolgreiche Reformstrategien in verschiedenen westeuropäischen Ländern erkennen. So wurden in Österreich, Deutschland und den Niederlanden die mit den Reformen von 1988, 1990 bzw. 1995 gesetzlich eingeführten neuen ambulanten Sanktionen jeweils zuvor in der Praxis im Rahmen von Modellprojekten systematisch erprobt. Die durch empirische Begleitforschung bestätigte Praktikabilität und Akzeptanz der Projekte war Voraussetzung für die landesweite Umsetzung des Reformprogramms. Der Prozess der Erprobung und der Schaffung von Akzeptanz vor allem bei Staatsanwälten und Richtern braucht Zeit und bedarf einer kontinuierlichen Aufbauarbeit und Fortbildung, die in sozialen Umbruchsituationen wie derzeit in Mittel- und Osteuropa schwierig zu gestalten ist. Dennoch erscheint eine "Jugendstrafrechtsreform durch die Praxis" (vgl. für Deutschland Bundesministerium der Justiz 1989) gegenüber einer Reform „von oben“, die häufig die Bereitstellung einer entsprechenden Infrastruktur versäumt, vorzugswürdig.

Die Entwicklung in den mittel- und osteuropäischen Ländern ist geprägt durch einen deutlichen Anstieg der registrierten Jugendkriminalität seit Ende der 1980er bis Mitte der 1990er Jahre. Die in allen Ländern anerkannte Notwendigkeit einer Reform des Jugendstrafrechts ergibt sich aus dem politischen Anspruch, altes (häufig sowjetisches oder sowjetisch beeinflusstes) Recht zu ersetzen und den (west-)europäischen Standards, wie sie in den Grundsätzen des Europarats und der Vereinten Nationen zum Ausdruck gelangen, Geltung zu verschaffen. Dabei werden allerdings teilweise unterschiedliche kriminalpolitische Tendenzen sichtbar.

Die Reform hat in Legislative und Praxis seit Anfang der 1990er Jahre eine dynamische Entwicklung genommen, erkennbar nicht nur an zahlreichen Praxisprojekten, sondern auch an der Einsetzung von Gesetzesreform- 
kommissionen und - teilweise bereits - der Verabschiedung von Reformgesetzen (vgl. z. B. Estland, Litauen, Rumänien, Serbien, Slowenien, die Tschechische Republik). ${ }^{7}$

Zum einen steht der Aufbau einer eigenständigen Jugendgerichtsbarkeit im Vordergrund (vgl. z. B. die Baltischen Staaten, Kroatien, die Tschechische Republik, Slowakei, Russland) und damit im Zusammenhang die Entwicklung rechtsstaatlicher Verfahrensstrukturen unter Berücksichtigung der speziellen erzieherischen Anliegen bei jungen Rechtsbrechern. Allerdings existieren beispielsweise in den baltischen Staaten, Rumänien und in Russland bislang keine eigenständigen Jugendgerichte und fehlen weitgehend die infrastrukturellen Voraussetzungen für eine Einführung moderner sozialpädagogischer Konzepte im Bereich der Jugendhilfe. Erst in einigen Modellregionen wird der Aufbau einer selbständigen Jugendgerichtsbarkeit erprobt (z. B. in Russland in Rostow/Don und in Rumänien in Brasov, vgl. Părosanu in Dünkel/Grzywa/Pruin 2009).

Zum anderen werden im Rechtsfolgenbereich neben dem für notwendig erachteten Ausbau von ambulanten Erziehungsmaßnahmen einschließlich der Diversion auch harte Sanktionen freiheitsentziehender Art propagiert, um insbesondere Wiederholungstäter oder junge Gewalttäter abzuschrecken. Dementsprechend führt die noch weitgehend fehlende Infrastruktur und teilweise auch fehlende Akzeptanz ambulanter Sanktionen zu einer nach wie vor häufigen Anwendung freiheitsentziehender Sanktionen. Allerdings zeigt die Entwicklung beispielsweise in Russland, dass ein Zurückgehen auf frühere Sanktionsmuster (mit einem Anteil von Freiheitsstrafen von ca. 50\%) nicht erfolgt und insbesondere Formen der Strafaussetzung zur Bewährung die freiheitsentziehenden Sanktionen quantitativ übertreffen. Deutlich wird in allen mittel- und osteuropäischen Ländern, dass das Prinzip des Freiheitsentzugs als „ultima ratio“ ernster genommen und stationäre Sanktionen zunehmend zurückgedrängt werden.

Im Bereich der ambulanten Sanktionen werden die Schwierigkeiten der Einrichtung der dafür notwendigen Infrastruktur deutlich. So fehlte es zunächst weitgehend an methodisch qualifizierten Sozialarbeitern bzw. Sozialpädagogen, zumal die entsprechenden Ausbildungsgänge vielfach erst im Aufbau sind.

Sowohl in den mittel- und osteuropäischen wie den westeuropäischen Ländern haben sich Elemente einer "restorative justice" durchgesetzt. In allen Reformgesetzen der letzten 15 Jahre spielen der Täter-OpferAusgleich, die mediation, oder Sanktionen wie die Wiedergutmachung bzw. Entschuldigung beim Opfer eine besondere Rolle. Zumeist gehen diese Tendenzen mit informellen Verfahrenserledigungen einher (Diversion). Zum Teil werden sie wie in England/ Wales (reparation, restitution order) oder Deutschland (Wiedergutmachungsauflage, Täter-Opfer-Ausgleich als erzieherische Weisung, vgl. $\mathbb{S} \mathbb{1 0}, 15 \mathrm{JGG}$ ) als eigenständige Sanktionen des Jugendgerichts vorgesehen. Die in Neuseeland erprobten ,family group conferences", ein mediativer Ansatz unter Einbeziehung und Aktivierung des sozialen Familiennetzwerks von Opfern und Tätern, sind nunmehr in Belgien - zunächst noch als Modellprojekte - eingeführt worden. Die aktuelle Jugendstrafrechtsreform in Nordirland (Juvenile Justice [Northern Ireland] Act von 2002) legt ebenfalls einen Schwerpunkt auf diesen Ansatz von Familienkonferenzen, die seit 2003 in Form der sog. youth conferences erprobt werden. Ferner wurde die in England/Wales 1998 eingeführte reparation order (Wiedergutmachungsauflage) im neuen Gesetz in Nordirland übernommen (vgl. i. E. O’Mahony/Campbell 2006).

Inwiefern diese Elemente der „restorative justice" wirklich die Sanktionspraxis insgesamt verändern oder nur als Alibi oder „Feigenblatt" eines eher repressiven Jugendstrafrechts anzusehen sind, kann nur vor dem Hintergrund der jeweiligen Länder mit ihren unterschiedlichen Traditionen entschieden werden. Für die deutsche jugendgerichtliche Sanktionspraxis kann man ebenso wie für die österreichische sagen, dass der Täter-Opfer-Ausgleich einen auch quantitativ beachtlichen Stellenwert erlangt hat (bei Jugendlichen ca. 8\% aller Sanktionen, vgl. Dünkel/Scheel/Schäpler 2003 für das Bundesland Mecklenburg-Vorpommern; zu Österreich vgl. Jesionek 2001; Bruckmüller 2006). Nimmt man noch die gemeinnützige Arbeit als im weiteren Sinne "restorative“ Sanktion hinzu, so werden mehr als ein Drittel aller Jugendlichen und Heranwachsenden von derartigen im Idealfall, d. h. wenn sie pädagogisch begleitet werden, als „konstruktive" Alternativen des Jugendstrafrechts $\mathrm{zu}$ bezeichnenden Sanktionen erfasst (vgl. hierzu auch Heinz 2008; Dünkel 2006).

\section{Altersgrenzen strafrechtlicher Verantwortlichkeit und Typologie von Jugendstrafrechtssystemen im europäischen Vergleich}

Vergleicht man die Jugendstrafrechtssysteme idealtypisch, so kann man nach wie vor die beiden „klassischen“ Orientierungen des Justiz- und des Wohlfahrtsmodells unterscheiden. ${ }^{8}$ Das Wohlfahrtsmodell ist charakterisiert durch ein weites Entscheidungsermessen des Jugendrichters oder anderer Entscheidungsträger (Sozialarbeiter, Psychologen etc.), tendenziell zeitlich unbestimmte Sanktionen, deren Beendigung vom eingeschätzten Erziehungserfolg abhängt und informellen Verfahren ohne ausgeprägte verfahrensrechtliche Garantien. Anknüpfungspunkte erzieherischer Interventionen sind typischerweise straffälliges ebenso wie auffälliges Verhalten (z. B. „Verwahrlosung“, „Gefährdung “; am stärksten ausgeprägt in dieser Richtung sind die Jugendrechtssysteme in Belgien, Polen und Schottland).

Demgegenüber knüpft das Justizmodell ausschließlich an straffälligem Verhalten entsprechend der allgemeinen Strafgesetze an. Die Reaktionen sind tatschuldproportional und zeitlich bestimmt. Das Verfahren sieht tendenziell die gleichen Garantien wie das Erwachsenenstrafverfahren vor, Entscheidungen werden in einem förmlichen Verfahren von (dem Anspruch nach spezialisierten) Juristen getroffen.

Diese idealtypischen Modelle existieren allerdings selten in „Reinform“, häufig finden sich Mischtypen wie z. B. das deutsche Jugendstrafrecht mit einer Verzahnung rein jugendhilferechtlicher Erziehungsmaßnahmen mit einem Justizmodell, das verfahrensrechtlich die Garantien und Grundsätze des Erwachsenenstrafverfahrens weitgehend übernimmt, und im Sanktionenbereich den Vorrang des Erziehungsgedankens bei nur ausnahmsweise $\mathrm{zu}$ verhängenden echten Kriminalstrafen (in Deutschland: Jugendstrafe gem. \17 JGG) propagiert.

Man kann die Tendenzen einer minimalistischen Intervention (Vorrang informeller Verfahren einschließlich des Täter-OpferAusgleichs) sowie von Wiedergutmachungsstrategien im Rahmen einer Typologie des Jugendstrafrechts auch als eigenständige Modelle begreifen (,minimum intervention model“, "restorative justice model", vgl. Cavadino/Dignan 2006, S. 199 ff., 205 ff.). Neben dem „minimum intervention model“ (Vorrang von Diversion und ambulanten Sanktionen) und „restorative justice model“ (Vorrang konfliktschlichtender Reaktionen) 
nennen Cavadino und Dignan (2006, S. $210 \mathrm{ff}$.) noch das bereits erwähnte "neocorrectionalist model ", das insbesondere die aktuellen Tendenzen in England und Wales charakterisiert (siehe oben).

Auch hier fällt es schwer, klare Abgrenzungen zu finden, denn die meisten kontinentaleuropäischen Jugendkriminalrechtssysteme haben sowohl die jugendhilfe- und justizorientierte (rechtsstaatliche) Philosophie, wie auch Elemente der „minimalen Intervention“ (so vor allem die Praxis in Deutschland, vgl. Dünkel 2006), der „restorative justice“ als auch des „neo-correctionalism“ (z. B. Prinzip der stärkeren Verantwortlichmachung des Täters und der Eltern, härtere Strafen bei Widerholungstätern, geschlossene Unterbringung bei Kindern etc.) aufgenommen. Unterschiede zeigen sich eher in der starken oder weniger starken Orientierung an „restorativen“ oder bestrafenden Elementen.

Sehr gut kommt dieser "neue Mix“ in den 2003 verabschiedeten Empfehlungen des Europarats über „New ways of dealing with juvenile offenders" zum Ausdruck (vgl. die Recommendation R [2003] 20). Auf der einen Seite wird betont, dass sich der Vorrang der Diversion und minimalistischer Interventionen bewährt habe und deshalb für die „normale“, episodenhafte Jugendkriminalität daran festzuhalten sei. Hierbei habe sich insbesondere auch die Integration von Wiedergutmachungsstrategien (Täter-Opfer-Ausgleich u. ä.) als positive Entwicklung ausgezeichnet. Zugleich fordert die Empfehlung aber auch die stärkere Inpflichtnahme von Gewalt- und Wiederholungstätern und von deren Eltern. Elemente des „neo-correctionalism " werden deutlich, wenn etwa die Betonung auf Frühintervention und Prävention von Jugenddelinquenz einerseits und effektive Sanktionierung andererseits gelegt wird, die nach den Erkenntnissen „what works, with whom, under what circumstances" wissenschaftlich begründet erfolgen soll (vgl. Recommendation R (2003) 20 und unten 5.). Andererseits enthält die neue Empfehlung des Europarats klassisch rechtsstaatliche Empfehlungen, wenn etwa die strikte Begrenzung von Polizei- und Untersuchungshaft gefordert wird. Der Erziehungsund Resozialisierungsgedanke wird neben der Prävention an zweiter Stelle als Leitprinzip anerkannt. Daraus folgen im einzelnen verschiedene Maßnahmen wie z. B. die Orientierung an der Wiedereingliederung vom ersten Tag einer Haftstrafe an (vgl. Nr. 19 der Recommendation), der überleitungso- rientierten Vollzugsgestaltung über Hafturlaub, Freigang und Übergangshäuser etc. (vgl. Nr. 20). Nicht zuletzt das dritte Prinzip der Recommendation, die stärkere Berücksichtigung von Opferinteressen, spiegelt die im Jugendstrafrecht besonders entwickelten Ansätze der Wiedergutmachung etc. wider.

Zusammenfassend kann man die europäischen Jugendkriminalrechtssysteme weithin als Mischsysteme aus jugendhilfe- und jugendstrafrechtlichen Elementen ansehen, mehr oder weniger ergänzt durch die oben genannten neuen Orientierungen. ${ }^{9}$ Insoweit ist trotz der nicht zu verleugnenden nationalen Besonderheiten eine beachtliche Konvergenz der Systeme zu erkennen. Das rein jugendhilferechtliche System ist, insbesondere infolge der 1989 verabschiedeten Kinderrechtskonvention der Vereinten Nationen (vgl. hierzu Höynck/Neubacher/ Schüler-Springorum 2001), international vergleichend gesehen auf dem Rückzug. Dies zeigt sich weniger in Europa, wo es ohnehin in einer mehr oder weniger deutlichen „Reinform“ nur noch in Belgien, Polen und Schottland existiert, als beispielsweise in Lateinamerika. ${ }^{10}$

Obwohl man insgesamt gesehen von einer europäischen Philosophie des Jugendstrafrechts sprechen kann, die im Hinblick auf die auch in den Empfehlungen des Europarats von 2003 zum Ausdruck gelangenden Prinzipien der Erziehung/Resozialisierung, der Einbeziehung von Opferbelangen durch mediation und Wiedergutmachung und der Beachtung rechtsstaatlicher Verfahrensgarantien deutlich wird, ist eine Harmonisierung des altersbezogenen Anwendungsbereichs bislang nicht ersichtlich.

Der Beginn strafrechtlicher Verantwortlichkeit liegt bei 10 (England), 12 (Niederlande), 13 (Frankreich), 14 (Deutschland, Italien, Österreich, Spanien und zahlreiche mittelund osteuropäische Länder), 15 (die skandinavischen Länder) oder gar 16 Jahren (bei bestimmten Delikten in Russland und anderen osteuropäischen Ländern). Als häufigste Altersgrenze der Strafmündigkeit kann man nach den aktuellen Reformen in Mittel- und Osteuropa das Alter von 14 Jahren erkennen (vgl. die nachfolgende Tabelle 1).

Teilweise werden rein erzieherische Reaktionen oder besser Sanktionen des Familienoder Jugendgerichts auch schon ab einem früheren Alter zugelassen, wie etwa neuerdings explizit in Frankreich ab 10 und in Griechenland ab 8 Jahren. In der Schweiz sind nach dem seit Anfang 2007 geltenden
Recht für 10- bis 14-Jährige nur Erziehungsmaßnahmen vorgesehen, die Verhängung einer Jugendstrafe ist erst ab 15 Jahren möglich. Zum Teil existiert ferner eine abgestufte Strafmündigkeit, indem nur für schwerere Delikte die Strafmündigkeit ab 14, im Übrigen ab 16 Jahren vorgesehen wird (Litauen, Russland, Slowenien).

$\mathrm{Ob}$ mit diesen beträchtlichen Unterschieden tatsächlich erhebliche Unterschiede in der Sanktionspraxis korrelieren, erscheint nicht immer eindeutig. Denn die im reinen Erziehungsrecht als „ultima ratio“ mögliche Unterbringung in einem (im schlechtesten Fall geschlossenen) Erziehungsheim kann unter Umständen in ihrer Eingriffsintensität und zeitlichen Dauer einer Unterbringung im Jugendstrafvollzug gleichkommen. Auch besagen die gesetzlichen Altersgrenzen nicht notwendig etwas über eine mehr oder weniger punitive Orientierung der Jugendstrafrechtspflege bzw. der Jugendhilfe aus. Die Praxis unterscheidet sich häufiger deutlich von der in Reformdiskursen vorgetragenen Rhetorik (vgl. hierzu Doob/Tonry 2004, S. 16 ff.). Dementsprechend sind Verschärfungen in Gesetzen manchmal Folge veränderter Praxis, manchmal tragen sie dazu bei, und in einigen Ländern wie insbesondere Deutschland gibt es trotz z. T. massenmedial anders vermittelter Bedrohungsszenarien auch eine erstaunliche Stabilität in der Praxis der Jugendstrafrechtspflege.

Im Bereich der oberen Altersgrenzen (d. h. bis zu welchem Alter Jugendstrafrecht oder jugendstrafrechtliche Sanktionen anwendbar sind) gibt es interessante Tendenzen, die Anwendung des Jugendstrafrechts oder zumindest seiner spezifisch erzieherischen Rechtsfolgen auf die Gruppe der 18-20-jährigen Heranwachsenden auszudehnen, wie dies schon 1953 in Deutschland erfolgt ist (vgl. die Reformen in den letzten Jahren in den Niederlanden, Kroatien, Österreich und Litauen). ${ }^{11}$

Die jugendkriminologisch gut belegte Begründung liegt in den verlängerten Übergangsphasen (im soziologischen Sprachgebrauch: „Statuspassagen“) von der Jugend als Lebensphase ins Erwachsenenleben. So haben sich Ausbildungszeiten und die Integration in das berufliche Erwerbsund familiäre Erwachsenenleben (mit der Gründung einer eigenen Familie etc) in den letzten 50 Jahren weit über das 20 . Lebensjahr hinaus verlängert. Damit sind entwicklungspsychologische „Krisensituationen" und Probleme beim Übergang ins 
Erwachsenenleben typisch für die Altersgruppe der Heranwachsenden, aber auch bis in die Mitte des dritten Lebensjahrzehnts hinein (vgl. hierzu Pruin 2007).

Die Praxis in Deutschland bezogen auf die Einbeziehung von Heranwachsenden in die Jugendgerichtsbarkeit und ihre flexible nach der Entwicklungsreife erfolgende Sanktionierung nach Jugend- oder Erwachsenenstrafrecht (vgl. \105 JGG) kann als großer Erfolg für die Weiterentwicklung einer ei- genständigen Jugendgerichtsbarkeit angesehen werden. Seit 1953 hat sich der Anteil von nach Jugendstrafrecht abgeurteilten Heranwachsenden auf insgesamt über 60\% erhöht, bei der besonders schweren Kriminalität liegt er bei über $90 \%$. Die Folge sind in diesem Bereich mildere Strafen als nach Erwachsenenstrafrecht, das bis zu 15 Jahre Freiheitsstrafe oder lebenslange Freiheitsstrafe androht, während im Jugendstrafrecht die Höchststrafe 10 Jahre beträgt. Die Tendenz, gerade bei den besonders schweren Delikten
Jugendstrafrecht anzuwenden, erklärt sich aus den im deutschen Erwachsenenstrafrecht geltenden hohen Mindeststrafrahmen von z. B. drei oder 5 Jahren beim schweren Raub, oder 1-2 Jahren bei bestimmten (schweren) Drogendelikten oder bei Vergewaltigung/sexueller Nötigung. Die Jugendrichter wollen damit unangemessen lange und erzieherisch abträgliche Freiheitsstrafen vermeiden. Erwachsenenstrafrecht wird bei Heranwachsenden dagegen bevorzugt bei bestimmten leichteren Delikten wie Straßen-

Tabelle 1: Vergleich der Altersgrenzen strafrechtlicher Verantwortlichkeit und der Altersgruppen im Jugendstrafvollzug bzw. vergleichbaren Einrichtungen in Europa

\begin{tabular}{|c|c|c|c|}
\hline Land & Strafmündigkeitsalter & $\begin{array}{r}\text { Alter, ab dem Erwach- } \\
\text { senenstrafrecht angewendet } \\
\text { werden kann/muss }\end{array}$ & Altersgruppen im Jugendstrafvollzug o. ä. \\
\hline Belgien & $16 * * * * * * * / 18$ & $16 / 18$ & -18 (nur Erziehungsheime) \\
\hline Bulgarien & 14 & 18 & $14-21$ \\
\hline Dänemark* & 15 & $15 / 18 / 21$ & $15-23$ \\
\hline Deutschland & 14 & $18 / 21$ & $14-24$ \\
\hline England/Wales & $10 / 12 / 15 * *$ & 18 & $10 / 15-21$ \\
\hline Estland & 14 & 18 & 14-21 \\
\hline Finnland* & 15 & $15 / 18$ & $15-21$ \\
\hline Frankreich & $10 * * * * * * 13$ & 18 & $13-18+6$ Mon. $/ 23$ \\
\hline Griechenland & $8 * * * * * * / 13$ & $18 / 21$ & $13-21 / 25$ \\
\hline Irland & $12 / 16^{* *}$ & 18 & $10 / 12 / 16-18 / 21$ \\
\hline Italien & 14 & $18 / 21$ & $14-21$ \\
\hline Kroatien & $14 / 16^{* *}$ & $18 / 21$ & $14-21$ \\
\hline Lettland & 14 & 18 & $14-21$ \\
\hline Litauen & $14 * * * * 16$ & $18 / 21$ & $14-21$ \\
\hline Montenegro & $14 / 16^{* *}$ & $18 / 21$ & $14-23$ \\
\hline Niederlande & 12 & $16 / 18 / 21$ & $12-21$ \\
\hline Nordirland & 10 & $17 / 18 / 21$ & $10-16 / 17-21$ \\
\hline Norwegen* & 15 & 18 & $15-21$ \\
\hline Österreich & 14 & $18 / 21$ & $14-27$ \\
\hline Polen & $13 * * * * *$ & $15 / 17 / 18$ & $13-18 / 15-21$ \\
\hline Portugal & $12 * * * * / 16$ & $16 / 21$ & 12/16-21 \\
\hline Rumänien & $14 / 16$ & $18 /(20)$ & $16-21$ \\
\hline Russland & $14 * * * * 16$ & $18 / 21$ & $14-21$ \\
\hline Schweden* & 15 & $15 / 18 / 21$ & $15-25$ \\
\hline Schweiz & 10 & $18 / 25$ & $10-22 / 17-25 / 30$ \\
\hline Schottland & $8 * * * * * 16$ & $16 / 21$ & $16-21$ \\
\hline Serbien & $14 / 16^{* *}$ & $18 / 21$ & $14-23$ \\
\hline Slowakei & $14 / 15$ & $18 / 21$ & $14-18$ \\
\hline Slowenien & $14 * * * * / 16$ & $18 / 21$ & $14-23$ \\
\hline Spanien & 14 & 18 & $14-21$ \\
\hline Tschechien & 15 & $18 / 18+$ & $15-19$ \\
\hline Türkei & 12 & $15 / 18$ & $12-18 / 21$ \\
\hline Ukraine & $14 * * * * / 16$ & $18 / 21$ & 14-21 \\
\hline Ungarn & 14 & 18 & $14-24$ \\
\hline Zypern & 14 & $16 / 18 / 21$ & $14-21$ \\
\hline
\end{tabular}

* Nur Strafmilderungen im allg. Strafrecht

$*$ Bestrafungsmündigkeit - Jugendstrafvollzug;

$* * * \quad$ Nur für Straßenverkehrsdelikte

$* * * * \quad$ Nur für einige besonders schwere Delikte

$* * * * \quad$ Anwendung des Jugendhilferechts, keine strafrechtliche Verantwortlichkeit i. e. S.

$* * * * * \quad$ Nur erzieherische Strafen (sanctions éducatives) 
verkehrsdelikten (Fahren ohne Fahrerlaubnis, Trunkenheitsfahrten u. ä.) angewandt, weil im Erwachsenenstrafverfahren Delikte der Massendelinquenz sich routinemäßig im schriftlichen Strafbefehlsverfahren erledigen lassen (vgl. zusammenfassend Dünkel 2003a; 2006; Pruin 2007).

Diese Entwicklung in Deutschland steht in auffälligem Gegensatz zu Tendenzen in den USA, wo Jugendliche, teilweise auch Kinder, bei schweren Delikten vermehrt den Erwachsenengerichten überstellt werden, um so eine härtere Bestrafung als üblicherweise durch das Jugendgericht zu erreichen (vgl. zusammenfassend Stump 2003).

In den anderen europäischen Ländern, die eine Anwendung jugendstrafrechtlicher Sanktionen bei Heranwachsenden ermöglichen, wie z. B. die Niederlande, Litauen, Kroatien, Serbien, Slowenien oder Russland, ist die Praxis offenbar (noch) sehr zurückhaltend (vgl. zusammenfassend Pruin 2007). In den Niederlanden (dort werden lediglich ca. 1\% der Heranwachsenden mit jugendstrafrechtlichen Sanktionen belegt, vgl. Dünkel 2003a, S. 22) dürfte dies damit zusammenhängen, dass auch das niederländische Erwachsenenstrafrecht (im Gegensatz zum deutschen StGB, das als Alternativen zur Freiheitsstrafe ohne Bewährung praktisch nur die Geldstrafe und die Freiheitsstrafe mit Bewährung kennt) eine Fülle von alternativen Sanktionen, insbesondere die gemeinnützige Arbeit als Hauptsanktion, vorsieht, so dass ein "Ausweichen“ in das Jugendstrafrecht nicht unbedingt nahe liegt oder notwendig erscheint. Sicherlich spielt aber in Deutschland eine entscheidende Rolle, dass die Regelung des \$105 JGG schon seit 1953 existiert und, dass in jedem Fall die Jugendgerichte für die Aburteilung von Heranwachsenden zuständig sind (vgl. \108 JGG), die eine „natürliche“ Tendenz entwickelt haben, überwiegend das ihnen vertrautere JGG anzuwenden.

Im Bereich der jugendstrafrechtlichen Rechtsfolgen lassen sich europaweit einerseits Tendenzen einer Ausweitung der Diversion erkennen, jedoch wird diese auch häufiger mit erzieherischen oder schlicht normverdeutlichenden Inhalten verknüpft, wie dies am Beispiel des Täter-Opfer-Ausgleichs besonders deutlich wird. So hat Griechenland im Rahmen einer Strafprozessreform 2003 für unter 18-Jährige weit reichende Möglichkeiten der Durchbrechung des Legalitätsprinzips, insbesondere in Verbindung mit Wiedergutmachungsbemühungen des Täters, geschaffen (Art. 45a grStPO, vgl. Spinellis/Tsitsoura 2006) und auch das neue tschechische (2003) und das serbische Jugendstrafrecht (von 2006) favorisieren die Diversion in diesem Sinn (vgl. Válková 2006; Skulic 2009). Andererseits lassen sich z. B. in England/Wales neuerdings Einschränkungen erkennen, indem mit einer Reform von 1998 im Falle einer zweiten Auffälligkeit nach einer vorangegangenen „reprimand“ (Verwarnung) nur noch ein "final warning" möglich ist, bei weiteren Auffälligkeiten aber regelmäßig eine Anklage erfolgen muss (vgl. Cavadino/Dignan 2002, S. 286 ff.).

Dennoch kann man davon ausgehen, dass unabhängig von der stärker justiz- oder wohlfahrtsorientierten Ausgestaltung der Jugendstrafrechtssysteme der ganz überwiegende Teil der Jugendkriminalität außergerichtlich im Wege informeller Reaktionen (Diversion) erledigt wird. In Deutschland betraf dies $200668 \%$ in den alten und sogar $78 \%$ aller anklagefähigen Verfahren in den neuen Bundesländern (bundesweit: 69\%, vgl. Heinz 2008; Dünkel 2006). In Belgien, einem der wenigen Länder mit einem eindeutig wohlfahrtsrechtlich organisierten Jugendrecht werden ca. 80\% der Verfahren informell erledigt (vgl. van Dijk/Dumortier/ Eliaerts 2006; Drenkhahn 2006).

Ferner sind sozialpädagogisch konstruktive Maßnahmen wie Soziale Trainingskurse (Deutschland) oder sog. Arbeits- und Lernstrafen bzw. -projekte (Niederlande) erfolgreich implementiert worden. In vielen Ländern wird explizit der Erziehungsgedanke (Portugal) bemüht, im übrigen geht es - in der Sprache weniger ,aufgeladen“ - schlicht um Legalbewährung bzw. Spezialprävention (so auch die Empfehlung des Europarats über „New ways of dealing with juvenile delinquency ..." aus dem Jahr 2003).

Der internationale Vergleich belegt - abgesehen vielleicht von England/Wales - gerade keine Abkehr vom Erziehungsgedanken. Begrenzungen des Erziehungsstrafrechts durch Tatproportionalität bzw. Verhältnismäßigkeitsüberlegungen werden andererseits insbesondere bzgl. freiheitsentziehender Sanktionen betont.

Die Frage, ob angesichts neuer Kriminalitätsformen und Tätergruppen andere, ggf. härtere Sanktionen notwendig erscheinen, ist damit eindeutig mit "nein“ zu beantworten. Weder die Kriminalitätsentwicklung noch die vermeintliche Ineffizienz herkömmlicher erzieherischer bzw. spezialpräventiv begründeter Maßnahmen zwingen zu einer härteren Gangart im Bereich des Jugendkriminalrechts. Vielmehr kann als international belegt gelten, dass eingriffsschwächere Sanktionen des Jugendstrafrechts, eingeschlossen die Diversion, ggf. in Verbindung mit Täter-Opfer-Ausgleich, Wiedergutmachung und anderen sozialkonstruktiven Reaktionen, die Integration des ,normalen“ Jugenddelinquenten besser fördern als eingriffsintensivere, insbesondere freiheitsentziehende Sanktionen. ${ }^{12}$

\section{Die Empfehlungen des Europarats über "New ways of dealing with juvenile delinquency and the role of juvenile justice”}

Die Empfehlungen des Europarats über „New ways of dealing with juvenile delinquency and the role of juvenile justice" (siehe im Internet unter www.coe.int) von 2003 verfolgen als vorrangige Ziele:

\section{Die Vorbeugung von Kriminalität und Rückfall,}

2. Die Resozialisierung und Wiedereingliederung von Straftätern und

3. Die Berücksichtigung der Bedürfnisse und Interessen von Verbrechensopfern.

Der strategische Ansatz beinhaltet folgende Perspektiven:

Das Jugendstrafrechtssystem ist als Teilsystem in einer breiteren gemeindeorientierten Strategie zur Vorbeugung von Jugendkriminalität zu sehen, die den Kontext von Familie, Schule, Nachbarschaft und Gleichaltrigengruppe, in dem Jugendkriminalität entsteht, berücksichtigt (Nr. 2 der Recommendation). Die Ressourcen der Jugendstrafrechtspflege sollen auf schwere, gewalttätige und wiederholte Jugendkriminalität (serious, violent and persistent offending), ggf. in Verbindung mit Alkohol- und Drogenproblemen, konzentriert werden (Nr. 3). Es müssen geeignetere und effektivere Maßnahmen der Vorbeugung und Wiedereingliederung auch für junge Migranten, gruppenorientierte Täter, junge Mädchen und noch nicht Strafmündige entwickelt werden (Nr. 4).

Sanktionen sollten soweit möglich auf wissenschaftlichen Erkenntnissen der Wirkungsforschung beruhen (what works, with whom and under what circumstances, Nr. 5).

Besondere Aufmerksamkeit der Jugendkriminalpolitik ist den Konsequenzen für ethnische Minderheiten zu widmen, deshalb 
sollen die Verantwortlichen zu sog. impact statements verpflichtet werden (Nr. 6).

Als „neue Antworten“ schlägt die Recommendation vor:

Die Ausweitung der Diversion sollte fortgesetzt werden, dabei ist die Tatproportionalität (der Verhältnismäßigkeitsgrundsatz) zu wahren und die Freiwilligkeit des Täters zu beachten (Nr. 7). Bezogen auf schwere, gewalttätige und wiederholte Jugendkriminalität sollten ambulante (verhältnismäßige) Sanktionen fortentwickelt werden (ggf., soweit nicht kontraproduktiv, auch unter Einbeziehung der Eltern in die Verantwortlichkeit), insbesondere auch auf Wiedergutmachung und Aussöhnung mit dem Opfer ausgerichtete Maßnahmen (Nr. 8, $10)$.

Die Empfehlung für einen Ausbau ambulanter Sanktionen bei schwerer Kriminalität erscheint insofern bemerkenswert, als in diesem Bereich häufig von der Notwendigkeit freiheitsentziehender Sanktionen ausgegangen wird. Erfahrungen mit der Strafaussetzung zur Bewährung und mit ambulanten Erziehungs-/Behandlungsprogrammen im Rahmen der Bewährungshilfe oder Jugendhilfe haben aber gezeigt, dass auch mit strafrechtlich vorbelasteten, mit gewalttätigen oder in Gruppen agierenden jungen Straftätern erfolgreich gearbeitet werden kann (vgl. Dünkel 2003, S. 89 ff., 96 ff.). Insoweit ist es gelungen, Jugendliche ambulant zu betreuen, die man vor 20 oder 30 Jahren als klassisches Klientel des (Jugend-)Strafvollzugs betrachtet hat.

In Anbetracht der verlängerten Ausbildungszeiten und Statuspassagen ins Erwachsenenleben sollen die Sanktionen des Jugendstrafrechts bei Heranwachsenden entsprechend der Reifeentwicklung angewandt werden können (Nr. 11). Dies entspricht den genannten positiven Erfahrungen in Deutschland und aktuellen Gesetzesreformen z. B. in Litauen, Österreich und Spanien (s. o.)

Im Übrigen betonen die Empfehlungen an verschiedenen Stellen, die Notwendigkeit von auf wissenschaftlicher Grundlage erfolgenden Interventionen (risk asessment, evidence based interventions) und der empirischen Evaluation. Obwohl auch „neokorrektionalistische" Gedanken (z. B. bzgl. der Haftung von Eltern) anklingen, bleibt die Empfehlung Rec (2003) 20 der Tradition eines gemäßigten Erziehungsstrafrechts (Stichwort: minimum intervention), das auch bei schwerwiegenderer Kriminalität auf ambulante Sanktionen setzt, verhaftet.
Dies sollte gegenreformatorischen (repressiven) Reformforderungen als Merkposten entgegengehalten werden.

Die Implementation der Empfehlungen soll unter Beachtung von Qualitätsstandards in enger Zusammenarbeit der örtlichen Träger der Prävention und Intervention erfolgen. Ein ständiges „monitoring“ wie die Verbreitung (dissemination) guter Praxismodelle gehören ebenfalls zu den neuen Strategien.

Es bleibt abzuwarten, inwiefern die Empfehlungen die Reformpraxis in Europa, insbesondere auch in den mittel- und osteuropäischen Ländern beeinflussen werden. Leider ist zu vermuten, dass es für eine wissenschaftliche Evaluation häufig an der finanziellen Grundlage fehlen wird. Die Bedeutung einer wissenschaftlich fundierten („evidence based“) Kriminalpolitik kann allerdings nicht hoch genug eingeschätzt werden. Nur so wird es gelingen, populistischen Tendenzen, wie sie in Österreich von der Partei eines Jörg Haider, in Italien eines Silvio Berlusconi und in Franreich eines Le Pen auch mit Auswirkungen auf das Jugendstrafrecht propagiert werden, wirksam entgegenzutreten.

\section{Die Empfehlungen des Europarats für Jugendliche im Vollzug ambu- lanter und freiheitsentziehender Sanktionen von 2008 ("European Rules for Juveniles Subject to Sanctions and Measures")}

Im Januar 2006 verabschiedete das Ministerkomitee des Europarats die Neufassung der Europäischen Strafvollzugsgrundsätze (European Prison Rules, abgekürzt EPR, vgl. Council of Europe 2006; in deutscher Übersetzung: vgl. Bundesministerium der Justiz Berlin u. a. 2007). Zugleich setzte das Komitee für Kriminalitätsprobleme (Committee on Crime Problems, CDPC) eine weitere Expertenkommission ein, die Mindestgrundsätze des Europarats für inhaftierte Jugendliche und Jugendliche unter ambulanten Maßnahmen erarbeiten sollte. Der Arbeitsauftrag bezog sich ausdrücklich auf den stationären und ambulanten Bereich und geht schon insofern über die EPR hinaus. Aber auch, was den Freiheitsentzug gegenüber Jugendlichen anbelangt, gehen die neuen Regeln weiter als die EPR: es soll nämlich jegliche Form der Freiheitsentziehung erfasst werden, und das bedeutet neben dem „klassischen“ Jugendstrafvollzug die Unterbringung in (ggf. geschlossenen) Erziehungsheimen und in psychiatrischen Anstalten (bzw. anderen Einrichtungen des
Maßregelvollzugs) sowie nicht zuletzt allen Formen des vorläufigen Freiheitsentzugs in Heimen oder in der Untersuchungshaft.

Die vom Europarat eingesetzte Expertenkommission, der Andrea Baechtold/Bern, Frieder Dünkel/Greifswald und Dirk van Zyl Smit/Nottingham angehörten, hat bis zum April 2008 die entsprechenden Mindestgrundsätze erarbeitet. Das CDPC hat in seiner Sitzung im Juni 2008 die Grundsätze abschließend beraten, die voraussichtlich Ende 2008 vom Ministerkomitee des Europarats beschlossen werden. Die Arbeitsergebnisse sind im Internet unter www.coe. int auf der Seite des Penological Council (PC-CP) einzusehen. Im Folgenden wird ein Überblick über die Grundzüge der zukünftigen Empfehlungen gegeben.

Die Empfehlungen für den Vollzug ambulanter und stationärer Sanktionen werden in 7 Hauptteile gegliedert sein: ebenso wie bei den EPR wird es einen ersten Teil mit allgemeinen Grundsätzen geben („Basic Principles"), die sowohl für ambulante wie für alle Formen stationärer Sanktionen gelten. Der zweite Hauptteil betrifft ambulante, der dritte stationäre Sanktionen. In den weiteren Teilen geht es übergreifend um „Legal advice and assistance”, d. h. die Frage des Zugangs zu Rechtsbeiständen u. ä., im fünften Teil um Beschwerden, Rechtsmittel, Inspektionen und das „Monitoring“. Ein weiterer Hauptteil (Part VI.) betrifft das Personal und Teil VII. die Evaluation bzw. (Begleit-)Forschung sowie die Zusammenarbeit mit den Medien und der Öffentlichkeit. Eine abschließende Regel (Part VIII.) fordert die regelmäßige Anpassung und Überarbeitung der Empfehlungen.

Vom Umfang her am umfangreichsten und am stärksten untergliedert sind die Teile 2 und 3, zumal im dritten Teil neben einem Allgemeinen Teil spezielle Teile für Jugendliche in Einrichtungen der Jugendhilfe, des Jugendstrafvollzugs und der (Jugend-)Psychiatrie sowie in Einrichtungen der vorläufigen Freiheitsentziehung (vorläufige Heimerziehung und Untersuchungshaft) vorgesehen sind.

Ebenso wie bei den EPR versucht der vorliegende Entwurf von Empfehlungen bzw. Mindestgrundsätzen im Rahmen von „Basic Principles“ wesentliche Inhalte, die für den Gesamtbereich ambulanter und freiheitsentziehender Sanktionen von besonderer Bedeutung sind, „vor die Klammer“ zu ziehen und damit eine grundsätzliche Orientierung zu geben. 
Als generelle Zielsetzung wird den Empfehlungen in der Präambel Folgendes voran gestellt: „The aim of the present Rules is to uphold the rights and safety of juvenile offenders subject to sanctions or measures and to promote their physical, mental and social well-being when subjected to community sanctions and measures or any form of deprivation of liberty.

Nothing in these Rules ought to be interpreted as precluding the application of other relevant international human rights instruments and standards that are more conducive to ensuring the rights, care and protection of juveniles. In particular, the provisions of Recommendation Rec(2006)2 on the European Prison Rules and of Recommendation $R$ (92) 16 on the European Rules on Community Sanctions and Measures shall be applied to the benefit of juvenile offenders in as far as they are not in conflict with these Rules."

Damit wird deutlich gemacht, dass die vorliegenden Empfehlungen nicht hinter anderen bereits existierenden Menschenrechtsstandards zurückbleiben wollen. Man könnte daraus den allgemeinen Grundsatz des Verbots der Schlechterstellung von Jugendlichen gegenüber Erwachsenen ableiten.

Die 20 als „Basic Principles“ formulierten Grundsätze lauten wie folgt:

1. Juvenile offenders subject to sanctions or measures shall be treated with respect for their human rights.

2. The sanctions or measures that may be imposed on juveniles as well as the manner of their implementation shall be specified by law and based on the principles of social integration and education and on the prevention of re-offending.

3. Sanctions and measures shall be imposed by a court or if imposed by another legally recognised authority they shall be subject to prompt judicial review. They shall be determinate and imposed for the minimum necessary period and only for a legitimate purpose.

4. The minimum age for the imposition of sanctions or measures as a result of the commission of an offence shall not be too low and shall be determined by law.

5. The imposition and implementation of sanctions or measures shall be ba- sed on the best interests of the juvenile offenders, limited by the gravity of the offences committed (principle of proportionality) and take account of their age, physical and mental well-being, development, capacities and personal circumstances (principle of individualisation) as ascertained when necessary by psychological, psychiatric or social inquiry reports.

6. In order to adapt the implementation of sanctions and measures to the particular circumstances of each case the authorities responsible for the implementation shall have a sufficient degree of discretion without leading to serious inequality of treatment.

7. Sanctions or measures shall not humiliate or degrade the juveniles subject to them.

8. Sanctions or measures shall not be implemented in a manner that aggravates their afflictive character or poses an undue risk of physical or mental harm.

9. Sanctions or measures shall be implemented without undue delay and only to the extent and for the period strictly necessary (principle of minimum intervention).

10. Deprivation of liberty of a juvenile shall be a measure of last resort and imposed and implemented for the shortest period possible. Special efforts must be undertaken to avoid pre-trial detention.

11. Sanctions or measures shall be imposed and implemented without discrimination on any ground such as sex, race, colour, language, religion, sexual orientation, political or other opinion, national or social origin, association with a national minority, property, birth or other status (principle of non-discrimination).

12. Mediation or other restorative measures shall be encouraged at all stages of dealing with juveniles.

13. Any justice system dealing with juveniles shall ensure their effective participation in the proceedings concerning the imposition as well as the implementation of sanctions or measures. Juveniles shall not have fewer legal rights and safeguards than those provided to adult offenders by the general rules of criminal procedure.
14. Any justice system dealing with juveniles shall take due account of the rights and responsibilities of the parents and legal guardians and shall as far as possible involve them in the proceedings and the execution of sanctions or measures, except if this is not in the best interests of the juvenile. Where the offender is over the age of majority the participation of parents and legal guardians is not compulsory. Members of the juveniles' extended families and the wider community may also be associated with the proceedings where it is appropriate to do so.

15. Any justice system dealing with juveniles shall follow a multi-disciplinary and multi-agency approach and be integrated with wider social initiatives for juveniles in order to ensure an holistic approach to and continuity of the care of such juveniles (principles of community involvement and continuous care).

16. The juvenile's right to privacy shall be fully respected at all stages of the proceedings. The identity of juveniles and confidential information about them and their families shall not be conveyed to anyone who is not authorised by law to receive it.

17. Young adult offenders may, where appropriate, be regarded as juveniles and dealt with accordingly.

18. All staff working with juveniles perform an important public service. Their recruitment, special training and conditions of work shall ensure that they are able to provide the appropriate standard of care to meet the distinctive needs of juveniles and provide positive role models for them.

19. Sufficient resources and staffing shall be provided to ensure that interventions in the lives of juveniles are meaningful. Lack of resources shall never justify the infringement of the human rights of juveniles.

20. The execution of any sanction or measure shall be subjected to regular government inspection and independent monitoring.

Grundsatz Nr. 1 betont, dass Jugendliche, die staatlichen Sanktionen ausgesetzt sind, die Achtung sämtlicher Menschenrechte genießen. Ebenso wie die EPR geht es demgemäß nicht nur um die Achtung der Men- 
schenwürde, sondern um den Kanon sämtlicher Grundrechte.

Grundsatz Nr. 2 bezieht sich auf den Grundsatz der Erziehung bzw. Wiedereingliederung als alleinigem Ziel sowohl bei der Verhängung als auch beim Vollzug ambulanter und freiheitsentziehender Sanktionen. Generalpräventiven oder allein sichernden Strafzwecken wird im Kommentar zu den Basic Principles klarstellend eine eindeutige Absage erteilt. Im Hinblick auf die in einzelnen Ländern bestehende Möglichkeit, lebenslange Freiheitsstrafe zu verhängen oder eine zeitlich unbestimmte Verwahrung im Sinn der Sicherungsverwahrung auch für Jugendliche vorzusehen, muss folgender Satz aus dem Kommentar zu denken geben: "The rule on social integration would therefore not allow long-term security measures or life sentences that aim solely to protect society from young offenders and do not give them the prospect of release within a reasonable period."

Grundsatz Nr. 3 betrifft das aus rechtsstaatlicher Sicht selbstverständliche Prinzip, dass Sanktionen grundsätzlich von einem Gericht auferlegt werden und - soweit dies einer anderen Institution übertragen wird - der Rechtsweg unmittelbar eröffnet sein muss. Dies könnte von Bedeutung sein, wenn z. B. das nationale Recht vorsieht, dass ein Bewährungshelfer die Rahmenbedingungen der Bewährungsaufsicht bzw. Weisungen oder Auflagen ändern kann. Von grundlegender Bedeutung ist die Feststellung in Rule 3, dass alle Sanktionen und Maßnahmen von bestimmter Dauer sein müssen, zeitlich unbestimmte Strafen und Maßnahmen also ausgeschlossen sein sollen. Die Verhängung lebenslanger Freiheitsstrafen und die in Deutschland derzeit diskutierte nachträgliche Sicherungsverwahrung für Jugendliche würde diesem Grundsatz ebenso wenig entsprechen wie die Möglichkeit, im ambulanten Sanktionsbereich die Entziehung der Fahrerlaubnis oder Formen der Bewährungsaufsicht lebenslänglich anzuordnen. Auf internationaler Ebene nicht neu ist der Grundsatz, dass Sanktionen entsprechend dem Verhältnismäßigkeitsprinzip nur für eine möglichst kurze Dauer angeordnet werden sollen („,for the minimum necessary period“). In Basic Principle Nr. 3 geht es um die Anordnung von Sanktionen, während Grundsatz Nr. 9 den gleichen Gedanken in Bezug auf den Vollzug von Sanktionen wiederholt. Grundsatz Nr. 10 hebt das Prinzip der möglichst geringfügigen und kurzen Eingriffsintensität für die Anordnung (Ulti-
ma-ratio-Prinzip) und den Vollzug freiheitsentziehender Maßnahmen nochmals besonders hervor (s. u.)

Grundsatz Nr. 4 bezieht sich auf die Untergrenze des Alters strafrechtlicher Verantwortlichkeit. Ähnlich wie die Beijing-Rules der Vereinten Nationen und der existierenden Instrumente des Europarats enthält sich die vorliegende Empfehlung angesichts der Heterogenität der geltenden Altersgrenzen in Europa (vgl. oben Tabelle 1) einer bestimmten Festlegung und bleibt mit der Formulierung, dass das Alter der Strafmündigkeit „nicht zu niedrig“ angesetzt werden solle, eher unverbindlich, ist aber doch als klares und kritisches Signal an Länder wie England und Wales oder Irland bzw. die Schweiz zu sehen, die mit dem Mindestalter von 10 Jahren deutlich vom europäischen Durchschnitt von ca. 14 Jahren abweichen. Im Kommentar wird darüber hinaus darauf verwiesen, dass Länder mit einem relativ niedrigen Strafmündigkeitsalter darauf achten sollten, ggf. die Bestrafungsmündigkeit bzgl. freiheitsentziehender Sanktionen i. S. von Jugendstrafvollzug höher anzusetzen. Dies ist in der Schweiz auch der Fall, wo eine Freiheitsstrafe i. S. der deutschen Jugendstrafe erst ab dem Alter von 15 Jahren möglich ist.

Grundsatz Nr. 5 betrifft den spezifisch jugendhilferechtlichen Gedanken, dass alle Maßnahmen unter dem Leitmotiv des Kindeswohls stehen sollen. Der Kommentar verweist in diesem Zusammenhang explizit auf außerjustizielle Konfliktlösungsformen i. S. der „restorative justice“, die dem Kindeswohl häufig am ehesten entsprechen dürften. Ferner wiederholt Basic Principle Nr. 5 den auch schon in früheren Europaratsempfehlungen deutlich gewordenen Konsens, dass einerseits individualisierte, erzieherische bzw. spezialpräventive Zwecke verfolgende Sanktionen zu verhängen sind, diese aber durch das Prinzip der Tatproportionalität begrenzt werden müssen und demgemäß das Maß der Tatschuld überschreitende Sanktionen unzulässig sind (vgl. ähnlich die Rules Nr. 8 und 13 der Empfehlung [2003] 20 des Europarats). Im Kommentar wird hervorgehoben, dass das Prinzip der Individualprävention voraussetzt, dass differenzierte Diagnosen und Erziehungsvorschläge auf der Basis von ausführlichen Berichten (,social inquiry reports", die über die deutsche Praxis der Jugendgerichtshilfeberichte hinausgehen) jedenfalls dann erstellt werden müssen, wenn es sich nicht um Bagatelldelinquenz handelt und im Rechtsfolgenbereich ein- griffsintensivere als Diversionsmaßnahmen angezeigt erscheinen.

Grundsatz Nr. 6 betont das bei der Sanktionswahl und -bemessung notwendige Maß an Flexibilität bzw. Ermessen, jedoch darf dies nicht zu einer regional oder in anderer Hinsicht erheblichen Ungleichbehandlung junger Straftäter führen.

Grundsatz Nr. 7 untersagt jegliche Form erniedrigender Behandlung sowohl im ambulanten (z. B. bei der Art und Ausgestaltung gemeinnütziger Arbeit) wie auch im stationären Bereich (bei der Art der Unterbringung in überfüllten Anstalten etc.).

Grundsatz Nr. 8 untersagt zusätzliche, über die mit der Sanktion zwangsläufig verbundenen Einschränkungen hinausgehende Übelszufügungen, die den Wesensgehalt der Sanktion unnötig verschärfen. Hier dürften einige osteuropäische Rechtsordnungen mit der Ausgestaltung des Jugendstrafvollzugs nach unterschiedlichen Schweregraden in der Tradition einer schulddifferenzierenden Vollzugsgestaltung Probleme haben.

Grundsatz Nr. 9 hebt - wie oben erwähnt eine weitere Dimension des Verhältnismäßigkeitsprinzips hervor, dass nämlich der Vollzug von Sanktionen nicht länger als notwendig, d. h. möglichst kurz sein soll (Prinzip der „minimalen Intervention“). Dementsprechend müssen sowohl im ambulanten wie im stationären Bereich der vorzeitigen Beendigung von Maßnahmen bzw. Sanktionen vorgesehen werden, wenn der Zweck der Maßnahme vorzeitig erreicht ist oder eine vorzeitige Beendigung bzw. Entlassung verantwortet werden kann. Weiterhin betont Grundsatz Nr. 9, dass Maßnahmen möglichst ohne Verzögerungen vollzogen werden sollen (,without undue delay“), was ihren erzieherischen Charakter stärken soll.

Grundsatz Nr. 10 wiederholt die Regelung in Nr. 37 der Kinderrechtskonvention, von Nr. 17 der Beijing-Rules ebenso wie die Empfehlungen des Europarats von 1987 und 2003, dass Freiheitsentzug - egal in welcher Form - „ultima ratio“ bleiben muss, von möglichst kurzer Dauer sein soll und nur für den gesetzlich vorgesehenen Zweck vollstreckt werden darf. Der Kommentar betont in diesem Zusammenhang, dass der Aufenthalt in einer Anstalt auch nicht aus erzieherischen Gründen über das als Minimum vertretbare Maß hinaus verlängert werden darf. Jedoch sollen die Einrichtungen dafür Sorge tragen, dass Jugendliche eine in der Institution begonnene Ausbildungsmaßnahme 
nach der Entlassung möglichst „nahtlos“ fortsetzen können.

Wie alle internationalen (und nationalen) Menschenrechtsstandards (vgl. z. B. Art. 14 EMRK, Nr. 13 EPR) heben auch die vorliegenden Empfehlungen in Grundsatz Nr. 11 das Verbot unzulässiger Diskriminierung wegen Geschlecht, Rasse, sexueller Orientierung, Religion usw. besonders hervor. Der Kommentar stellt hierzu klar, dass besondere Behandlungsmaßnahmen aufgrund eines festgestellten Förderbedarfs keine Diskriminierung bedeuten.

Grundsatz Nr. 12 hebt die Mediation bzw. Konfliktschlichtung zwischen Täter und Opfer hervor, die in jedem Stadium des Verfahrens und im Zusammenhang mit den vorliegenden Empfehlungen auch bei der Vollstreckung und dem Vollzug von Sanktionen stets besonders zu prüfen ist.

Grundsatz Nr. 13 betrifft die vorzusehende effektive Teilhabe von Jugendlichen im Verfahren ebenso wie bei der Vollstreckung von Sanktionen. Hierbei wird das Verbot der Schlechterstellung gegenüber Erwachsenen besonders betont. Der Kommentar benennt hierzu beispielhaft, dass auch Jugendliche in rein jugendhilferechtlichen Verfahren einen Anspruch haben, einen Rechtsbeistand in Anspruch nehmen zu können und, dass unabhängig von der Verfahrensart - immer dann, wenn eine freiheitsentziehende Sanktion möglich ist, ein Fall der notwendigen Verteidigung gegeben sein soll.

Grundsatz Nr. 14 betrifft die Beteiligung der Erziehungsberechtigten und - nach Möglichkeit - des sozialen Umfelds (, wider community“), die mit Ausnahme derjenigen Fälle, in denen dies dem Kindeswohl widersprechen würde, zu gewährleisten ist. Ein derartiger Ausschluss der Erziehungsberechtigten - so der Kommentar - ist durch Gutachten von Psychologen o. a. Sachverständigen zu begründen und formell von einem Gericht zu entscheiden bzw. zu begründen.

Grundsatz Nr. 15 greift die Notwendigkeit des bereits in den Empfehlungen des Europarats von 2003 hervorgehobenen multidisziplinären („multi-agency“) Ansatzes (vgl. Nr. 21 der Recommendation [2003] 20) im Bereich von Jugendhilfe- und Jugendstrafrecht auf, was im Grunde nichts anderes bedeutet, als dass Juristen, Sozialarbeiter, Psychologen, ggf. Psychiater, Lehrer und andere beteiligte Disziplinen und entsprechende Institutionen (Jugendamt, Bewährungshilfe, Schule etc.) zusammenarbeiten sollen, ein Prinzip, das in Deutschland insbesondere im
Bereich der Jugendgerichtshilfe gesetzlich verankert ist (vgl. $\$ 52$ SGB VIII; $\mathbb{\$}$ 38, 72a JGG). Die Empfehlung geht aber noch darüber hinaus, indem das Gemeinwesen bzw. die Jugendarbeit insgesamt als Integrationspartner benannt werden. Ferner wird der Grundsatz der durchgehenden Betreuung ("continuous care“) hervorgehoben, der vor allem bei den Übergängen von Untersuchungshaft zur Strafhaft und zur (bedingten) Entlassung im dritten Hauptteil zum Freiheitsentzug eine besondere Rolle spielt und dort nochmals aufgegriffen wird. Als Modell einer weitergehenden und intensivierten Kooperation von Polizei, Jugendhilfe und Justiz kann der in Deutschland entwickelte Ansatz eines Hauses des Jugendrechts angesehen werden. ${ }^{13}$

Grundsatz Nr. 16 bezieht sich auf den Persönlichkeits- bzw. Datenschutz, der auch auf die Erziehungsberechtigten erstreckt wird.

Grundsatz Nr. 17 greift die bereits in Nr. 11 der Recommendation (2003) 20 vorgeschlagene weitergehende Einbeziehung von Heranwachsenden in das Jugendstrafrecht auf. Der Kommentar zu den vorliegenden Empfehlungen betont, dass Heranwachsende sich angesichts verlängerter Ausbildungszeiten und verzögerter sozialer Reife heutzutage generell in einem Übergangsstadium befinden, das ihre rechtliche Gleichstellung mit Jugendlichen und eine mildere Sanktionierung im Vergleich zu älteren Erwachsenen rechtfertigt. Die meisten Länder haben dem Übergangsstadium, in dem sich auch Heranwachsende noch befinden, entweder durch eine Einbeziehung in das Jugendstrafrecht (bzw. der Anwendung jugendstrafrechtlicher Sanktionen) oder durch besondere Milderungsvorschriften im Rahmen des Erwachsenenstrafrechts Rechnung getragen (vgl. zusammenfassend Pruin 2007; Dünkel/Pruin 2008).

Grundsatz Nr. 18 ist Nr. 8 der EPR nachgebildet und hebt die besondere Rolle des Personals der Jugendhilfe und Jugendkriminalrechtspflege hervor. Dementsprechend bedarf es einer besonderen Ausbildung bzw. Qualifikation und Auswahl, ferner sind die materiellen Rahmenbedingungen für den Erfolg der Arbeit und insbesondere die Fortbildung sowie Supervision sicherzustellen.

Auch Grundsatz Nr. 19 lehnt sich inhaltlich an die EPR an (hier Nr. 4), indem die Mittelknappheit in keinem Fall als Rechtfertigung für Grundrechtseinschränkungen akzeptiert wird. Positiv formuliert Satz 1 von Grundsatz Nr. 17 (inhaltlich an Grundsatz Nr. 18 anschließend), dass ausreichende personelle Ressourcen vorzuhalten sind, die gewährleisten, dass die Maßnahmen gegenüber Jugendlichen hohen Qualitätsstandards entsprechen.

Grundsatz Nr. 20 entspricht Nr. 9 der EPR und hebt die Notwendigkeit eines umfassenden Systems staatlicher und unabhängiger Kontrolle und Aufsicht („independent inspection" und "monitoring“) für den Bereich der Vollstreckung bzw. des Vollzugs ambulanter und freiheitsentziehender Maßnahmen gegenüber Jugendlichen hervor. Dazu gehört neben einem effektiven Individualrecht auf gerichtliche Kontrolle einzelner Entscheidungen des Vollstreckungsbzw. Vollzugspersonals ein System von unabhängigen Inspektionen, ferner wird für stationäre Einrichtungen im Kommentar die Einführung von Ombudsleuten vorgeschlagen.

Auf die Detailvorschläge zur Vollstreckung ambulanter und zum Vollzug freiheitsentziehender Sanktionen kann aus Raumgründen nicht näher eingegangen werden (vgl. hierzu Dünkel/Baechtold/van Zyl Smit 2007; Dünkel 2008).

\section{Ausblick}

Das Jugendstrafrecht ist durch die Entwicklung der Jugendkriminalität der 1990er Jahre unter Druck geraten. Ein allein auf positive Spezialprävention ausgerichtetes Erziehungsstrafrecht gerät bei brutalen, ggf. fremdenfeindlichen oder rechtsextremen Gewalttätern in Legitimationsnot und Argumentationsschwierigkeiten angesichts einer massenmedial teilweise aufgeheizten Debatte über angeblich notwendige härtere und generalpräventiv zu begründende Strafen. Die Entwicklung der Jugendkriminalität in Europa gibt allerdings insgesamt gesehen keinen Anlass zu einer Kehrtwende in der Jugendkriminalpolitik. Nach wie vor ist Jugendkriminalität ganz überwiegend episodenhaft und durch eher bagatellhafte Delinquenz charakterisiert. Zwar ist nicht zu verkennen, dass ein kleiner Teil von maximal $5 \%$ der männlichen Jugendlichen einer Geburtskohorte, vor allem der frühauffälligen, durch erhebliche Desintegrationsphänomene Belasteten, in dauerhafte kriminelle Karrieren abzugleiten droht, jedoch ist auch und gerade bei diesen jungen Menschen ein gemäßigtes, und rechtsstaatlich begrenztes Jugendstrafrecht ausreichend und als effizienter zu bewerten als eine Orientierung an repressiven, insbesondere längerfristigen 
freiheitsentziehenden Sanktionen. Zudem werden auch diese „Karrieren“ Mitte des dritten Lebensjahrzehnts überwiegend abgebrochen. Zu Recht fordern die o. g. Empfehlungen des Europarats, gerade bei diesen schwierigen Fällen vermehrt ambulante Sanktionen von der Diversion bis hin zu spezialpräventiv Erfolg versprechenderen Alternativsanktionen im Vergleich zum Freiheitsentzug zu entwickeln und anzuwenden.

Eine Aufgabe des Erziehungsanspruchs oder - weniger pathetisch - der Spezialprävention als Leitmotiv des Jugendstrafrechts (vgl. \ 2 JGG i. d. F. v. 1.1.2008) würde zu einer ungerechtfertigten Verschärfung des Sanktionsniveaus führen und vor allem die Eigenständigkeit des Jugendstrafrechts als Sonderstrafrecht gefährden. Gleichwohl ist nicht zu verkennen, dass mit der Ausdifferenzierung des Sanktionenkatalogs im Erwachsenenstrafrecht einerseits und mit der Stärkung prozessualer Verfahrensgarantien für Minderjährige die Systeme teilweise konvergieren (vgl. z. B. die Niederlande). Dennoch sollte das Jugendstrafrecht mehr als nur ein gemildertes Erwachsenenstrafrecht sein und bleiben. Jugendliche sind aufgrund ihres entwicklungsbedingten Übergangsstatus anders zu behandeln als Erwachsene (Junger-Tas 2006, S. 510). Die breite Vielfalt erzieherischer Maßnahmen in den europäischen Jugendstrafrechtsordnungen mit einer Vernetzung mit der Jugendhilfe verdeutlicht dies eindrucksvoll. Die Empfehlungen des Europarats über neue Wege der Bekämpfung von Jugendkriminalität (2003) sowie für Jugendliche unter ambulanten und freiheitsentziehenden Maßnahmen (2008) stellen eine hilfreiche Orientierung für ein eigenständiges Jugendstrafrecht dar und entsprechen einem weitgehenden europäischen Konsens, auch in schwierigen Zeiten, eine rationale Kriminal- und Sozialpolitik für die Jugend zu bewahren. Ziel muss die Integration, nicht Ausgrenzung junger Rechtsbrecher sein. Diesem Ziel dienen konstruktive Maßnahmen wie die Mediation (Täter-Opfer-Ausgleich), soziale Kompetenzen verbessernde Erziehungshilfen und ein insgesamt besonnenes, rechtsstaatlich gemäßigtes Jugendstrafrecht.

Thesenartig lassen sich die hier dargelegten Befunde wie folgt zusammenfassen:

- Jugendkriminalität ist nach wie vor weniger besorgniserregend als manche Medien suggerieren.

- Der Anstieg der Jugendgewalt ist im Dunkelfeld weit weniger spektakulär als im Hellfeld, d. h. das Anzeigeverhalten hat sich verändert (vgl. u. a. BMI/BMJ 2006, 19 ff.).

- Im Übrigen verdienen Kinder und Jugendliche eher als Opfer denn als Täter der Gewalt die Aufmerksamkeit der Gesellschaft.

- Jugendkriminalität ist nach wie vor in aller Regel episodenhaft und weniger schwer.

- Gleichwohl bedarf es bei einer kleinen Gruppe erheblich und mehrfach Belasteter bzw. Benachteiligter der (u. U. frühzeitigen) normverdeutlichenden, aber vor allem auch stützenden bzw. fördernden Reaktion.

- Die klassischen Formen der Jugendhilfe und ambulanter Erziehungshilfen sind auch hier und bei jungen Gewalttätern zumeist ausreichend und erfolgreich.

- Wenn stationäre Unterbringung unerlässlich erscheint, sollte diese in einem erzieherischen (therapeutischen) Milieu (Heimerziehung oder sozialtherapeutischer Jugendstrafvollzug) stattfinden.

- Die Tendenzen der Jugendkriminalpolitik in Europa sind - soweit sie auf eine Ausweitung der erzieherischen Resozialisierungsangebote einschließlich einer Verstärkung der Wiedergutmachung (,restorative justice“) gerichtet sind - zu begrüßen.

- Insofern kommt den Empfehlungen des Europarats von 2003 und 2008 eine richtungsweisende Funktion zu.

- Jugend braucht Verständnis, Toleranz und Aufgeschlossenheit der Erwachsenen, um ihre Integrationsschwierigkeiten zu bewältigen.

- Ein repressives Jugendstrafrecht wäre insoweit eher kontraproduktiv.

- Abzulehnen sind damit neo-liberale, vorwiegend auf Bestrafung und Vergeltung ausgerichtete Ansätze (vgl. das ,neo-correctionalist model" in England/Wales), die keine empirisch begründbare Strategie einer rationalen Jugendkriminalpolitik darstellen.

- Formen der Restorative Justice (wiedergutmachende Strafrechtspflege) sind ein positiver Weg der Verantwortungsübernahme und gehen mit dem klassischen Erziehungsstrafrecht konform.

- Hauptaugenmerk sollte auf die (ggf. frühzeitige) Prävention und den Ausbau von Hilfemaßnahmen für Familien in prekärer sozioökonomischer und psychosozialer Lage gelegt werden.

- Noch immer gilt der Anfang des 20. Jahrhunderts von Franz von Liszt geprägte Grundsatz: die beste Kriminalpolitik ist eine gute Sozialpolitik.

\section{Literatur}

Albrecht, H.-J., Kilchling, M. (2002) (Hrsg.): Jugendstrafrecht in Europa. Freiburg i. Br.: Max-Planck-Institut für ausländisches und internationales Strafrecht.

Bailleau, F., Cartuyvels, Y. (2007): La Justice Pénale des Mineurs en Europe - Entre modèle Welfare et infléxions néo-libérales. Paris: L'Harmattan.

Bannenberg, B. (1993): Wiedergutmachung in der Strafrechtspraxis. Bonn: Forum-Verlag.

Bruckmüller, K. (2006): Austria: A Protection Model. In: Junger-Tas, J., Decker, S. H. (Hrsg.): International Handbook of Juvenile Justice. Dordrecht: Springer, S. 263-294.

Bundesministerium des Inneren, Bundesministerium der Justiz (2006): Zweiter Periodischer Sicherheitsbericht. Berlin: BMI/BMJ (auch im Internet veröffentlicht unter: www.BMI. de/Berichte

Bundesministerium der Justiz (1989) (Hrsg.): Jugendstrafrechtsreform durch die Praxis. Bonn: Bundesministerium der Justiz.

Bundesministerium der Justiz Berlin, Bundesministerium für Justiz Wien, Eidgenössisches Justiz- und Polizeidepartement (2007) (Hrsg.): Europäische Strafvollzugsgrundsätze. Die Empfehlung des Europarats Rec (2006) 2. Mönchengladbach: Forum Verlag Godesberg.

Council of Europe (1989): Social reactions to juvenile delinquency (Recommendation No. R (87) 20 and explanatory memorandum). Social reactions to juvenile delinquency among young people coming from migrant families (Recommendation No. R (88) 6. Strasbourg: Council of Europe, European Committee on Crime Problems.

Council of Europe (2003): New ways of dealing with juvenile delinquency and the role of juvenile justice. Recommendation (2003) 20. Strasbourg: Council of Europe (vgl. auch www.coe.int unter Ministerkomitee, Recommendations).

Cavadino, M., Dignan, J. (2006): Penal Systems. A Comparative Approach. London, Thousand Oaks, New Delhi: Sage.

Doob, A. N., Tonry, M. (2004): Varieties of Youth Justice. In: Tonry, M., Doob, A. N. (Hrsg.): Youth Crime and Justice. Chicago, London: University of Chicago Press (Crime and Justice Bd. 31), S. 1-20.

Drenkhabn, K. (2006): Rechtliche Reaktionsmöglichkeiten auf Kinderdelinquenz im europäischen Vergleich. Praxis der Rechtspsychologie 16 , Heft 1/2, S. 82-93.

Dünkel, F. (1997): Jugendstrafrecht in Europa Entwicklungstendenzen und Perspektiven. In: Dünkel, F., van Kalmthout, A., Schüler-Springorum, H. (Hrsg.): Entwicklungstendenzen und Reformstrategien des Jugendstrafrechts im europäischen Vergleich. Bonn: Forum Verlag Godesberg, S. 565-650.

Dünkel, F. (2003): Entwicklungen der Jugendkriminalität und des Jugendstrafrechts in Europa - ein Vergleich. In: Riklin. F. (Hrsg.): Jugendliche, die uns Angst machen. Was bringt das Jugendstrafrecht? Luzern: Caritas-Verlag, S. 50-124. 
Dünkel, F. (2003a): Heranwachsende im Jugendstrafrecht in Deutschland und im europäischen Vergleich. DVJJ-Journal 14, S. 19-27.

Dünkel, F. (2006): Juvenile Justice in Germany: Between Welfare and Justice. In: Junger-Tas, J., Decker, S. H. (Hrsg.): International Handbook of Juvenile Justice. Dordrecht: Springer, S. $225-262$.

Dünkel, F. (2008): Europäische Mindeststandards und Empfehlungen für jugendliche Straftäter als Orientierungspunkte für die Gesetzgebung und Praxis: die „European Rules for Juvenile Offenders Subject to Sanctions and Measures". In: Sonnen, B.-R. (Hrsg.): Dokumentation des 24. Deutschen Jugendgerichtstags. Mönchengladbach: Forum Verlag Godesberg (im Druck).

Dünkel, F., Baechtold, A., van Zyl Smit, D. (2007): Europäische Mindeststandards und Empfehlungen als Orientierungspunkte für die Gesetzgebung und Praxis - dargestellt am Beispiel der Empfehlungen für inhaftierte Jugendliche und Jugendliche in ambulanten Maßnahmen (die „Greifswald Rules"). In: Goerdeler, J., Walkenhorst, P. (Hrsg.): Jugendstrafvollzug in Deutschland. Neue Gesetze, neue Strukturen, neue Praxis? Mönchengladbach: Forum Verlag Godesberg, S. 114-140.

Dünkel, F., van Kalmthout, A., Schüler-Springorum, H. (1997) (Hrsg.): Entwicklungstendenzen und Reformstrategien im Jugendstrafrecht imeuropäischen Vergleich. Mönchengladbach: Forum Verlag Godesberg.

Dünkel, F., Drenkhahn, K. (2001): Behandlung im Strafvollzug: von „, nothing works“ zu „something works“. In: Bereswill, M., Greve, W. (Hrsg.): Forschungsthema Strafvollzug. Baden-Baden: Nomos Verlag, S. 387-417.

Dünkel, F., Gebauer, D., Geng, B., Kestermann, C. (2007): Mare-Balticum-Youth-Survey - Gewalterfahrungen von Jugendlichen im Ostseeraum. Mönchengladbach: Forum Verlag Godesberg.

Dünkel, F., Grzywa, J., Pruin, I. (2008) (Hrsg.): Juvenile Justice Systems in Europe - current situation, reform developments and good practices. Mönchengladbach: Forum Verlag Godesberg (in Vorbereitung).

Dünkel, F., Pruin, I. (2008): Young adult offenders in the criminal justice systems of European countries. In: Lösel, F., Bottoms, A., Farrington, D. (Hrsg.): Lost in Transition? Young adult offenders in the criminal justice system. Cullompton: Willan Publishing (im Druck).

Dünkel, F., Sakalauskas, G. (2001): Jugendstrafrecht und Jugendstrafrechtsreform in Litauen. DVJJ-Journal 12, S. 72-80.

Dünkel, F., Scheel, J., Schäpler, P. (2003): Jugendkriminalität und die Sanktionspraxis im Jugendstrafrecht in Mecklenburg-Vorpommern. Zeitschrift für Jugendkriminalrecht und Jugendhilfe, 14, S. 119-132.

Elsner, E., Molnar, H., Steffen, W. (2001): Kriminalität Heranwachsender und Jungerwachsener in München. München: Bayerisches Landeskriminalamt.

Estrada, F. (1999): Juvenile Crime Trends in PostWar Europe. European Journal on Criminal Policy and Research 7, S. 23-42.

Estrada, F. (2001): Juvenile Violence as Social Problem. British Journal of Criminology 41, S. 639-655.

Farrington, D., Langan, P., Tonry, M. (2004): Cross National Studies in Crime and Justice. Washington/DC: Bureau of Justice Statistics.

Feuerhelm, W., Kügler, N. (2003). Das „Haus des Jugendrechts" in Stuttgart Bad Cannstatt. Ergebnisse einer Evaluation. Mainz: Institut für Sozialpädagogische Forschung e. V.

Gabriel, G. (2004). Fast tracking and co-operation between police, justice and social services
- crime as a cause for looking if support is required. In: Centre for the Prevention of Youth Crime (Hrsg.): Prevention of Youth Crime in Germany: Educational Strategies. München: Deutsches Jugendinstitut, S. 99-112.

Garland, D. (2001): The Culture of Control. Crime and Social Order in Contemporary Society. Chicago: The University of Chicago Press.

Garland, D. (2001a) (Hrsg.): Mass Imprisonment. Social Causes and Consequences. London: Sage.

Graham, J. (1998): Aktuelle Entwicklungen in der Jugendjustiz in England und Wales. DVJJJournal 9, S. 317-321.

Haferkanmp, R. (2007): Neuere Entwicklungen im Jugendstrafrecht in Schweden und Finnland. RdJB 55, S. 167-190.

Heinz, W. (2002a): Entwicklung der Kriminalität junger Menschen - Anlass für eine Verschärfung des Jugendstrafrechts? DVJJ-Journal 13, S. 277-288.

Heinz, W. (2002b): Kinder- und Jugendkriminalität - ist der Strafgesetzgeber gefordert? Zeitschrift für die Gesamten Strafrechtswissenschaften 114, S. 519-583.

Heinz, W. (2004): Kriminalität in Deutschland unter besonderer Berücksichtigung der Jugendund Gewaltkriminalität. Internet-Publikation www.uni-konstanz.de/rtf/kik/Heinz_Kriminalitaet_in_Deutschland.htm

Heinz, W. (2008): Das strafrechtliche Sanktionensystem und die Sanktionierungspraxis in Deutschland 1882 - 2006 (Stand: Berichtsjahr 2006) Version: 1/2008. Internet-Publikation www.uni-konstanz.de/rtf/kis/sanks06.pdf.

Höynck, T., Neubacher, F., Schüler-Springorum, $H$. (2001): Internationale Menschenrechtsstandards und das Jugendkriminalrecht. Dokumente der Vereinten Nationen und des Europarats. Berlin: Forum Verlag Godesberg. Jensen, E., L., Jepsen, J. (2006) (Hrsg.): Juvenile Law Violators, Human Rights, and the Development of New Juvenile Justice Systems. Oxford, Portland/Oregon: Hart Publishing.

Jesionek, U. (2001): Das österreichische Jugendgerichtsgesetz. 3. Aufl., Wien: Juridica Verlag.

Junger-Tas, J. (2006): Trends in International Juvenile Justice - What Conclusions Can be Drawn? In: Junger-Tas, J., Decker, S. H. (2006) (Hrsg.): International Handbook of Juvenile Justice. Dordrecht: Springer, S. 505532.

Junger-Tas, J., Decker, S. H. (2006) (Hrsg.): International Handbook of Juvenile Justice. Dordrecht: Springer.

Kaiser, G. (1985): International vergleichende Perspektiven zum Jugendstrafrecht. In: Schwind, H.-D. u. a. (Hrsg.): Festschrift für G. Blau. Berlin, New York: Walter de Gruyter, S. 441457.

Kaiser, G. (1996): Kriminologie. Ein Lehrbuch. 3. Aufl. Heidelberg: C. F. Müller.

Kasten, A. (2003): Das französische Jugendstrafrecht. Die Auswirkungen der französischen Strafrechtsreform vom 9. September 2002 auf das französische Jugendstrafrecht. Zeitschrift für Jugendkriminalrecht und Jugendhilfe 1, S. 382-388.

Kerner, H.-J. (1993): Jugendkriminalität zwischen Massenerscheinung und krimineller Karriere. In: Nickolai, W., Reindl. R. (Hrsg.): Sozialarbeit und Kriminalpolitik. Freiburg i. Br.: Lambertus Verlag, S. 28-62.

Kerner, H.-J. (2001): Möglichkeiten und Grenzen der Prävention von Jugendkriminalität. In: Dölling, D. (Hrsg.): Das Jugendstrafrecht an der Wende zum 21. Jahrhundert. Berlin, New York: Walter de Gruyter, S. 99-124.

Kilchling, M. (2002): Vergleichende Perspektiven. In: Albrecht, H.-J., Kilchling, M. (Hrsg.): Jugendstrafrecht in Europa. Freiburg i. Br.:
Max-Planck-Institut für ausländisches und internationales Strafrecht, S. 475-532.

Kunkat, A. (2002): Junge Mehrfachauffällige und Mehrfachtäter in Mecklenburg-Vorpommern - eine empirische Untersuchung. Mönchengladbach: Forum Verlag.

Lösel, F. (2001): Behandlung oder Verwahrung? Ergebnisse und Perspektiven bei „psychopathischen " Straftätern. In: Rehn, G. u. a (Hrsg.): Behandlung „gefährlicher Straftäter". Pfaffenweiler: Centaurus, S. 36-53.

Lösel, F., Bender, D. (2000): Protektive Faktoren gegen Delinquenzentwicklungen. In: Jehle, J.-M. (Hrsg.): Täterbehandlung und neue Sanktionsformen. Mönchengladbach: Forum Verlag Godesberg, S. 117-153.

Lösel, F, Bliesener, T., Averbeck, M. (1998): Hat die Delinquenz von Schülern zugenommen? Ein Vergleich im Dunkelfeld nach 22 Jahren. DVJJ-Journal 9, S. 115-125.

Lösel, F, Bliesener, T. (2003): Aggression und Delinquenz unter Jugendlichen. Untersuchungen von kognitiven und sozialen Bedingungen. München, Neuwied: Luchterhand.

McCarney, W. (1996) (Hrsg.): Juvenile Delinquents and Young People in Danger in an Open Environment. Winchester.

Meblbye, J., Walgrave, L. (1998) (Hrsg.): Confronting Youth in Europe: Juvenile Crime and Juvenile Justice. Copenhagen: AKF Forlaget.

O'Mahony, D., Campbell, C. (2006): Mainstreaming Restorative Justice for Young Offenders through Youth Conferencing - The Experience of Northern Ireland. In: JungerTas, J., Decker, S. H. (Hrsg.): International Handbook of Juvenile Justice. Dordrecht: Springer, S. 93-115.

Patané, V. (2007) (Hrsg.): European Juvenile Justice Systems. First volume. Milano: Giuffrè Editore.

Pratt, J., u. a. (2005) (Hrsg.): The New Punitiveness. Trends, theories, perspectives. Cullompton: Willan Publishing.

Pruin, I. (2007): Die Heranwachsendenregelung im deutschen Jugendstrafrecht. Jugendkriminologische, entwicklungspsychologische, jugendsoziologische und rechtsvergleichende Aspekte. Mönchengladbach: Forum Verlag Godesberg.

Roberts, J., Hough, M. (2002) (Hrsg.): Changing Attitudes to Punishment. Public opinion, crime and justice. Cullompton: Willan Publishing.

Schwind, H.-D., u. a. (2001): Kriminalitätsphänomene im Langzeitvergleich am Beispiel einer deutschen Großstadt. Bochum 1975 - 1986 - 1998. Neuwied, Kriftel: Luchterhand.

Spinellis, C. D., Tsitsoura, A. (2006): The Emerging Juvenile Justice System in Greece. In: Junger-Tas, J., Decker, S. H. (Hrsg.): International Handbook of Juvenile Justice. Dordrecht: Springer, S. 309-324.

Stelly, W., u. a. (1998): Kontinuität und Diskontinuität sozialer Auffälligkeiten im Lebenslauf. MschrKrim 81, S. 104-122.

Stelly, W., Thomas, J. (2001): Einmal Verbrecher - immer Verbrecher? Wiesbaden: Westdeutscher Verlag.

Stoorgard, A. (2004): Juvenile Justice in Scandinavia. Journal of Scandinavian Studies in Criminology and Crime Prevention 5, S. 188-204.

Stump, B. (2003): „Adult time for adult crime“ - Jugendliche zwischen Jugend- und Erwachsenenstrafrecht. Mönchengladbach: Forum Verlag Godesberg.

Tiffer-Sotomayor, C. (2000): Jugendstrafrecht in Lateinamerika unter besonderer Berücksichtigung des Jugendstrafrechts in Costa Rica. Mönchengladbach: Forum Verlag.

Tiffer-Sotomayor, C., Llobet Rodríguez, J., Dünkel, F. (2002): Derecho Penal Juvenil. San José/Costa Rica: DAAD. 
Tonry, M. (2004): Punishment and Politics. Cullompton: Willan Publishing.

Tonry, M., Doob, A. N. (2004) (Hrsg.): Youth Crime and Justice. Chicago, London: University of Chicago Press (Crime and Justice Bd. 31).

Válková, H. (2006): Restorative Approaches and Alternative Methods: Juvenile Justice Reform in the Czech Republic. In: Junger-Tas, J., Decker, S. H. (Hrsg.): International Handbook of Juvenile Justice. Dordrecht: Springer, S. 377-395.

van Dijk, C., Dumortier, E., Eliaerts, C. (2006): Survival of the Protection Model? Competing Goals in Belgian Juvenile Justice. In: JungerTas, J., Decker, S. H. (Hrsg.): International Handbook of Juvenile Justice. Dordrecht: Springer, S. 187-223.

van Dijk, J., Manchin, R., van Kesteren, J, Nevala, S, Hideg. G. (2005): The Burden of Crime in the EU. Research Report: A Comparative Analysis of the European Crime and Safety Survey (EU ICS) 2005. Internet-Publikation http://www.europeansafetyobservatory.eu/ downloads/EUICS \%20-\%20The\%20Burden \%20of\%20Crime \%20in \%20the\%20EU.pdf 2005.

von Hofer, H. (2004): Crime and Reactions to Crime in Scandinavia. Journal of Scandinavian Studies in Criminology and Crime Prevention 5, S. 148-166.

Walter, M. (2005): Jugendkriminalität. 3. Aufl., Stuttgart u. a.: Richard Boorberg Verlag.

\section{Kontaktadresse:}

Prof. Dr. Frieder Dünkel, Ernst-MoritzArndt-Universität Greifswald, Rechts- und Staatswissenschaftliche Fakultät, Lehrstubl für Kriminologie, Domstr. 20, D-17487 Greifswald

Tel.: 03834-862138

E-mail:duenkel@uni-greifswald.de.

Internet: http://jura.uni-greifswald.de/duenkel.

\section{Fußnoten:}

1 Vgl. Kerner 1993; Elsner/Molnar/Steffen 2001; Heinz 2004; BMI/BMJ 2006; Dünkel/Gebauer/Geng/Kestermann 2007; zu Trends der Entwicklung der Jugendkriminalität in Europa vgl. Estrada 1999; 2001; zu entsprechenden Opferbefragungen vgl. van Dijk et al. 2005; BMI/BMJ 2006.

2 Vgl. Cavadino/Dignan 2002: 284 ff.; 2006: 215 ff.; Junger-Tas/Decker 2006; Bailleau/Cartuyvels 2007.

3 In Frankreich wurden Schwerpunkte insbesondere hinsichtlich der Verfahrensbeschleunigung und der härteren Reaktion gegenüber Mehrfach- bzw. Rückfalltätern gesetzt. So kann seit 2007 z. B. die zuvor übliche Strafmilderung bei Rückfalltätern entfallen, vgl. Kasten 2003; Casteignède/Pignoux in Dünkel/Gtrywa/Pruin 2009.
4 Vgl. zusammenfassend Dünkel 1997; 2003; Kilchling 2002; Cavadino/Dignan 2002, S. 284 ff.; 2006, S. 215 ff.; Junger-Tas/Decker 2006; Bailleau/Cartuyvels 2007.

5 Vgl. zusammenfassend von Hofer 2004; Stoorgard 2004; Haverkamp 2007.

6 Vgl. Pratt u. a. 2005; ferner gleichfalls vorwiegend mit Blick auf die Entwicklungen in den USA und England : Garland 2001; 2001a; Roberts/Hough 2002; Tonry 2004.

7 Vgl. z. B. Válková 2006; zusammenfassend Dünkel 2003, S. 69 ff.; Dünkel/Grzywa/Pruin 2009.

8 Vgl. Kaiser 1985; Dünkel 1997; 2003; Doob Tonry 2004, S. $1 \mathrm{ff}$.

9 Vgl. im einzelnen die Beiträge bei Dünkel/van Kalmthout/Schüler-Sprinorum 1997; Albrecht Kilchling 2002; Tonry/Doob 2004; Jensen/Jepsen 2006; Junger-Tas/Decker 2006; Bailleaul Cartuyvels 2007; Patané 2007.

$10 \mathrm{Vgl}$. Tiffer-Sotomayor 2000; Tiffer Sotomayor/ Llobet Rodríguez/Dünkel 2002.

11 Vgl. hierzu zusammenfassend Dünkel 2003, S. 82 ff.; 2003a; Pruin 2007; Dünkel/Pruin 2008.

12 Dies gilt im Übrigen auch für sog. Karrieretäter, vgl. Kerner 2001, S 111 ff., 124; hierzu ferner Stelly u. a. 1998; Lösel/Bender 2000; Stelly/ Thomas 2001.

$13 \mathrm{Zu}$ dem als erfolgreich eingeschätzten Mode in Stuttgart-Bad Cannstatt vgl. Feuerhelm/ Kügler 2003; Gabriel 2004. Inzwischen haben verschiedene weitere Bundesländer Pläne der Übernahme solcher Strukturen entwickelt.

\section{Realität oder Fiktion?}

\section{Die Darstellung der Justiz im Fernsehen}

Ruth Herz

Politiker haben sich längst in einer Wirklichkeit eingerichtet, die - wie man ohne Übertreibung sagen kann - von den Medien bestimmt wird. Minister und Parlamentsabgeordnete sprechen häufiger mit und durch das Fernsehen, direkt'zu den Bürgern als dass sie sich im Bundestag äußern, was sie dazu zwingt sich mediengerecht zu äußern. Sie wissen zum Beispiel, dass sie kurze Sätze bilden und dabei einige Schlüsselsätze formulieren müssen, die aus dem Zusammenhang herausgenommen werden können und dennoch zitierfähig bleiben, damit sie am Abend in den Kurznachrichten vorkommen. Ihre Gesprächspartner sind dann nicht ibre politischen Partner oder Gegner und auch nicht die Bürger, sondern die Journalisten. Soweit die Exekutive und Administrative Gewalt im Staat. Im Folgenden wird der Frage nachgegangen, wie es mit der dritten Gewalt, der Justiz bestellt steht. Findet auch die Justiz im Fernsehen statt? Hat sich der Staat so weit zurückgezogen und die Aufgabe der Bestätigung gemeinsamer Normen und Werte den Massenmedien, insbesondere dem Fernsehen, überlassen?

Der Gerichtssaal ist ein Ort an dem sich täglich menschliche Konflikte und Dramen abspielen. Es ist daher nicht überraschend, dass es im Fernsehen an Geschichten und Berichten aus dem Gerichtssaal nicht mangelt. Diese bieten sich dem Medium des Fernsehens mit ihrer Einheit von Zeit, Ort und Handlung geradezu an. Die Geschichten werden in den verschiedenen Fernsehformaten, insbesondere in Nachrichtensen- dungen, in Dokumentarfilmen, in den in Deutschland bekannten Gerichtsserien und in anderen semifiktionalen sowie in rein fiktionalen Sendungen dargestellt.

Zunächst soll auf diese verschiedenen Formate eingegangen werden. Sie sollen unter besonderer Berücksichtigung der zunehmend unscharf werdenden Grenzen zwischen ihnen analysiert und kommentiert werden, um die eingangs gestellte Frage nach der Stellung der Justiz in einer Demokratie beantworten zu können. Dabei ist die Einstellung der Richter sowie der Journalisten zu diesen Fragen, im Kontext des gegenwärtigen politischen Klimas in Deutschland von besonderer Bedeutung.

\section{II}

Millionen von Zuschauern saßen in den letzten Jahren täglich nachmittags vor den Fernsehschirmen und verfolgten die Forma- 